\title{
Tuning the Reactivity and Bonding Properties of Metal Square-Planar Complexes by the Substitution(s) on the Trans-Coordinated Pyridine Ring
}

\author{
Olga Dvořáčková ${ }^{1,2}$ and Zdeněk Chval ${ }^{*}$ \\ ${ }^{1}$ Faculty of Health and Social Sciences, University of South Bohemia, J. Boreckého 27, 370 \\ 11 České Budějovice, Czech Republic, \\ ${ }^{2}$ Faculty of Science, University of South Bohemia, Branišovská 1760, 37005 České \\ Budějovice, Czech Republic \\ *Corresponding Author: chval@jcu.cz; +420-389-037-612
}

SUPPORTING INFORMATION 

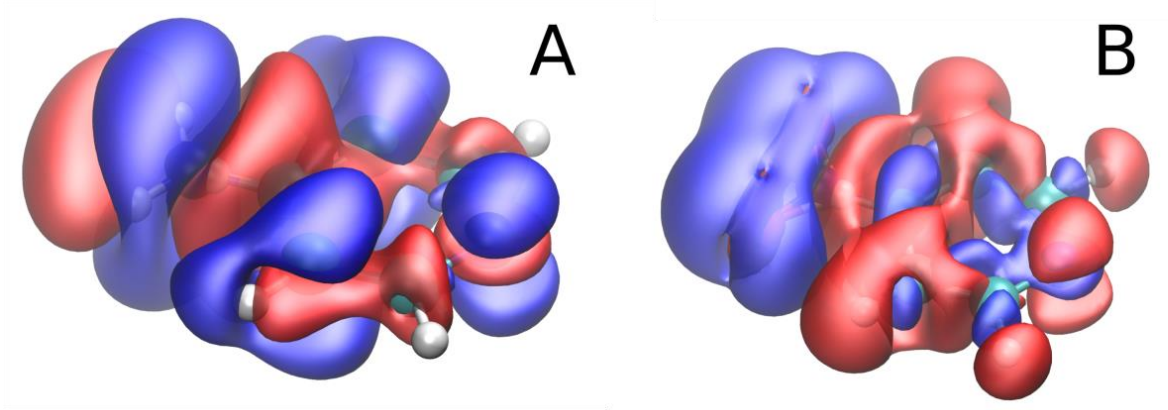

Figure S1: Electron density difference isosurfaces of $\mathbf{p}-\mathbf{N H}_{\mathbf{2}}(\mathrm{A})$ and $\mathbf{p}-\mathbf{N O}_{\mathbf{2}}(\mathrm{B})$ structures with respect to the reference pyrH structure which show electron accumulation (blue: 0.0004 a.u.) and depletion (red: -0.0004 a.u.) regions caused by $\mathrm{p}-\mathrm{NH}_{2}(\mathrm{~A})$ and $\mathrm{p}-\mathrm{NO}_{2}$ (B) substitution of the pyr ring. Electron densities were calculated on the pyrH geometry for all atoms of respective complexes except the $\mathrm{X}$ substituent atoms whose positions were optimized.

Table S1: Pt-pyrX interaction in X-R, X-TS and X-P structures: $\mathrm{Pt}-\mathrm{N}_{\text {pyrX }}$ bond lengths (in $\AA$ ); the total NPA charges of the Pt atom $(\mathrm{q}(\mathrm{Pt}))$ and the pyrX ligands $(\mathrm{q}(\mathrm{pyrX}))$; ETS-NOCV energy decomposition terms $\Delta E_{\text {Pauli }}, \Delta E_{\text {elst }}, \Delta E_{\text {orb }}, \Delta E_{\text {disp }}, \Delta E_{\text {orb }}^{\sigma}, \Delta E_{\text {orb }}^{\pi}$ were obtained at BLYP-D3BJ/TZ2P//B3LYP/BS1 level. $\Delta \mathrm{E}_{\text {Bind }}$ energy values were calculated at B3LYPD3BJ/BS2//B3LYP/BS1 level. All energy values are in $\mathrm{kcal} / \mathrm{mol}$.

\begin{tabular}{|l|l|r|r|r|r|r|r|r|r|r|r|}
\hline $\mathrm{X}-\mathrm{R}$ & & $\mathrm{Pt}-\mathrm{N}_{\mathrm{pyrX}}$ & $\mathrm{q}(\mathrm{Pt})$ & $\mathrm{q}(\mathrm{pyrX})$ & $\Delta E_{\text {Pauli }}$ & $\Delta E_{\text {elst }}$ & $\Delta E_{\text {orb }}$ & $\Delta E_{\text {disp }}$ & $\Delta E_{\text {orb }}^{\sigma}$ & $\Delta E_{\text {orb }}^{\pi}$ & $\Delta E_{\text {bind }}$ \\
\hline $\mathrm{H}$ & & 2.081 & 0.617 & 0.245 & 127.6 & -122.5 & -61.6 & -7.2 & -40.7 & -11.6 & -65.7 \\
\hline $\mathrm{DMA}$ & $\mathrm{o}-$ & 2.099 & 0.617 & 0.251 & 133.1 & -125.1 & -66.6 & -12.1 & -39.8 & -11.3 & -72.3 \\
\hline & $\mathrm{m}-$ & 2.077 & 0.617 & 0.261 & 131.9 & -130.7 & -65.1 & -7.6 & -42.8 & -10.6 & -73.3 \\
\hline & $\mathrm{p}-$ & 2.074 & 0.615 & 0.275 & 132.7 & -134.5 & -65.7 & -7.4 & -43.4 & -12.7 & -76.8 \\
\hline $\mathrm{NH}_{2}$ & $\mathrm{o}-$ & 2.086 & 0.601 & 0.260 & 135.6 & -129.9 & -64.5 & -9.0 & -41.2 & -10.9 & -69.9 \\
\hline & $\mathrm{m}-$ & 2.078 & 0.616 & 0.254 & 129.7 & -127.4 & -63.4 & -7.4 & -41.8 & -10.3 & -70.4 \\
\hline & $\mathrm{p}-$ & 2.077 & 0.612 & 0.268 & 130.5 & -131.0 & -64.0 & -7.3 & -42.5 & -12.1 & -73.8 \\
\hline $\mathrm{Br}$ & $\mathrm{o}-$ & 2.099 & 0.622 & 0.231 & 120.2 & -110.3 & -61.1 & -9.8 & -38.0 & -11.6 & -62.5 \\
\hline & $\mathrm{m}-$ & 2.083 & 0.617 & 0.238 & 124.1 & -115.2 & -62.0 & -7.4 & -40.2 & -11.8 & -62.1 \\
\hline & $\mathrm{p}-$ & 2.081 & 0.615 & 0.243 & 126.0 & -118.2 & -62.3 & -7.3 & -40.2 & -12.3 & -63.2 \\
\hline $\mathrm{SH}$ & $\mathrm{o}-$ & 2.088 & 0.615 & 0.233 & 131.4 & -122.6 & -64.9 & -9.4 & -39.7 & -13.1 & -67.5 \\
\hline & $\mathrm{m}-$ & 2.081 & 0.618 & 0.244 & 126.2 & -119.7 & -62.7 & -7.4 & -40.6 & -10.4 & -65.3 \\
\hline & $\mathrm{p}-$ & 2.079 & 0.614 & 0.252 & 127.5 & -122.1 & -63.4 & -7.3 & -41.0 & -12.6 & -67.0 \\
\hline $\mathrm{OH}$ & $\mathrm{o}-$ & 2.088 & 0.592 & 0.243 & 135.7 & -124.3 & -65.1 & -8.5 & -40.1 & -13.9 & -63.5 \\
\hline & $\mathrm{m}-$ & 2.080 & 0.617 & 0.243 & 127.6 & -123.5 & -61.9 & -7.3 & -40.6 & -11.7 & -66.8 \\
\hline
\end{tabular}




\begin{tabular}{|c|c|c|c|c|c|c|c|c|c|c|c|}
\hline & $\mathrm{p}-$ & 2.081 & 0.613 & 0.254 & 127.4 & -124.2 & -62.0 & -7.3 & -41.1 & -11.7 & -67.9 \\
\hline \multirow[t]{3}{*}{$\mathrm{F}$} & o- & 2.100 & 0.624 & 0.223 & 114.7 & -108.0 & -57.0 & -7.6 & -36.9 & -11.3 & -59.6 \\
\hline & $\mathrm{m}-$ & 2.084 & 0.616 & 0.234 & 124.3 & -116.6 & -60.5 & -7.2 & -39.5 & -11.7 & -61.4 \\
\hline & p- & 2.085 & 0.613 & 0.241 & 124.8 & -117.9 & -60.6 & -7.2 & -39.8 & -11.5 & -62.4 \\
\hline \multirow[t]{3}{*}{$\mathrm{Cl}$} & o- & 2.100 & 0.622 & 0.229 & 118.3 & -109.5 & -59.6 & -9.1 & -37.5 & -11.6 & -61.8 \\
\hline & m- & 2.084 & 0.615 & 0.236 & 123.8 & -115.3 & -61.3 & -7.3 & -39.8 & -11.7 & -61.8 \\
\hline & p- & 2.082 & 0.615 & 0.242 & 125.4 & -117.9 & -61.7 & -7.3 & -39.9 & -12.1 & -62.9 \\
\hline \multirow[t]{3}{*}{$\mathrm{CH}_{3}$} & o- & 2.087 & 0.616 & 0.253 & 131.1 & -124.3 & -63.1 & -9.6 & -40.9 & -11.7 & -67.8 \\
\hline & m- & 2.079 & 0.617 & 0.250 & 128.5 & -124.2 & -62.7 & -7.4 & -41.3 & -11.7 & -67.8 \\
\hline & p- & 2.079 & 0.617 & 0.252 & 128.6 & -124.9 & -62.7 & -7.3 & -41.4 & -11.9 & -68.4 \\
\hline \multirow[t]{3}{*}{$\mathrm{C} \equiv \mathrm{CH}$} & o- & 2.085 & 0.630 & 0.232 & 126.4 & -119.3 & -63.4 & -9.6 & -39.6 & -12.3 & -67.6 \\
\hline & m- & 2.082 & 0.619 & 0.242 & 125.4 & -118.2 & -62.3 & -7.4 & -40.3 & -10.6 & -64.3 \\
\hline & p- & 2.078 & 0.616 & 0.246 & 127.2 & -120.4 & -63.2 & -7.3 & -40.8 & -13.0 & -65.5 \\
\hline \multirow[t]{3}{*}{$\mathrm{NO}_{2}$} & o- & 2.111 & 0.613 & 0.205 & 114.4 & -103.3 & -57.7 & -10.2 & -34.7 & -11.6 & -57.5 \\
\hline & m- & 2.089 & 0.618 & 0.220 & 120.3 & -108.0 & -59.5 & -7.3 & -38.0 & -10.4 & -55.6 \\
\hline & p- & 2.084 & 0.618 & 0.221 & 122.8 & -109.6 & -60.6 & -7.2 & -38.6 & -12.7 & -57.5 \\
\hline \multicolumn{12}{|l|}{ X-TS } \\
\hline $\mathrm{H}$ & & 2.046 & 0.791 & 0.294 & 174.2 & -147.2 & -78.6 & -7.6 & -53.4 & -16.4 & -62.7 \\
\hline \multirow[t]{3}{*}{ DMA } & O- & 2.068 & 0.796 & 0.303 & 173.2 & -146.6 & -83.3 & -13.3 & -51.5 & -12.7 & -73.26 \\
\hline & m- & 2.042 & 0.788 & 0.314 & 179.4 & -155.8 & -82.8 & -8.0 & -56.3 & -11.1 & -70.70 \\
\hline & p- & 2.039 & 0.782 & 0.327 & 179.3 & -159.0 & -83.4 & -7.7 & -57.0 & -13.2 & -74.43 \\
\hline \multirow[t]{3}{*}{$\mathrm{NH}_{2}$} & o- & 2.050 & 0.770 & 0.312 & 192.7 & -160.6 & -85.9 & -9.8 & -57.4 & -16.0 & -67.1 \\
\hline & m- & 2.044 & 0.789 & 0.304 & 176.9 & -152.4 & -80.8 & -7.8 & -54.8 & -16.3 & -67.5 \\
\hline & $\mathrm{p}-$ & 2.043 & 0.784 & 0.319 & 177.0 & -155.4 & -81.5 & -7.7 & -55.7 & -16.7 & -71.1 \\
\hline \multirow[t]{3}{*}{$\mathrm{Br}$} & o- & 2.063 & 0.801 & 0.277 & 163.2 & -134.0 & -75.6 & -9.9 & -48.5 & -12.4 & -59.5 \\
\hline & m- & 2.049 & 0.793 & 0.286 & 170.5 & -140.0 & -78.6 & -7.8 & -52.1 & -12.6 & -58.9 \\
\hline & p- & 2.047 & 0.792 & 0.291 & 172.6 & -142.9 & -79.2 & -7.7 & -52.8 & -13.1 & -60.0 \\
\hline \multirow[t]{3}{*}{$\mathrm{SH}$} & o- & 2.051 & 0.785 & 0.273 & 163.2 & -134.0 & -75.6 & -9.9 & -48.5 & -12.4 & -64.3 \\
\hline & m- & 2.047 & 0.792 & 0.293 & 170.5 & -140.0 & -78.6 & -7.8 & -52.1 & -12.6 & -62.4 \\
\hline & $\mathrm{p}-$ & 2.045 & 0.789 & 0.302 & 172.6 & -142.9 & -79.2 & -7.7 & -52.8 & -13.1 & -64.0 \\
\hline \multirow[t]{3}{*}{$\mathrm{OH}$} & o- & 2.050 & 0.806 & 0.290 & 194.8 & -156.5 & -86.3 & -9.6 & -55.8 & -14.3 & -60.1 \\
\hline & m- & 2.046 & 0.792 & 0.293 & 173.6 & -147.7 & -78.7 & -7.6 & -53.1 & -12.4 & -63.7 \\
\hline & $\mathrm{p}-$ & 2.047 & 0.788 & 0.303 & 174.0 & -148.8 & -79.1 & -7.6 & -53.9 & -12.4 & -65.0 \\
\hline
\end{tabular}




\begin{tabular}{|c|c|c|c|c|c|c|c|c|c|c|c|}
\hline \multirow[t]{3}{*}{$\mathrm{F}$} & o- & 2.062 & 0.805 & 0.266 & 159.6 & -133.2 & -72.3 & -7.9 & -47.2 & -12.1 & -57.1 \\
\hline & m- & 2.050 & 0.794 & 0.282 & 170.4 & -141.1 & -76.9 & -7.6 & -51.6 & -12.5 & -58.2 \\
\hline & p- & 2.051 & 0.792 & 0.288 & 171.2 & -142.6 & -77.3 & -7.6 & -52.3 & -12.3 & -59.2 \\
\hline \multirow[t]{3}{*}{$\mathrm{Cl}$} & o- & 2.065 & 0.801 & 0.274 & 161.3 & -133.2 & -74.2 & -9.3 & -47.9 & -12.3 & -59.0 \\
\hline & $\mathrm{m}-$ & 2.050 & 0.793 & 0.284 & 170.1 & -140.0 & -77.9 & -7.7 & -51.6 & -12.5 & -58.7 \\
\hline & $\mathrm{p}-$ & 2.048 & 0.792 & 0.289 & 172.1 & -142.6 & -78.5 & -7.6 & -52.4 & -12.9 & -59.7 \\
\hline \multirow[t]{3}{*}{$\mathrm{CH}_{3}$} & o- & 2.054 & 0.788 & 0.305 & 175.1 & -147.5 & -79.4 & -10.0 & -53.2 & -12.1 & -66.3 \\
\hline & m- & 2.045 & 0.790 & 0.301 & 175.3 & -149.0 & -79.8 & -7.7 & -54.1 & -12.5 & -64.9 \\
\hline & p- & 2.044 & 0.789 & 0.302 & 175.1 & -149.5 & -79.9 & -7.6 & -54.2 & -12.6 & -65.5 \\
\hline \multirow[t]{3}{*}{$\mathrm{C} \equiv \mathrm{CH}$} & o- & 2.051 & 0.811 & 0.291 & 171.3 & -143.6 & -79.0 & -9.8 & -51.2 & -13.2 & -66.8 \\
\hline & m- & 2.048 & 0.792 & 0.291 & 171.4 & -142.7 & -79.0 & -7.7 & -52.5 & -11.2 & -61.4 \\
\hline & p- & 2.044 & 0.791 & 0.295 & 173.8 & -145.1 & -80.2 & -7.7 & -53.4 & -13.7 & -62.5 \\
\hline \multirow[t]{3}{*}{$\mathrm{NO}_{2}$} & o- & 2.072 & 0.799 & 0.243 & 159.6 & -128.2 & -73.2 & -10.7 & -45.6 & -16.4 & -54.5 \\
\hline & m- & 2.053 & 0.797 & 0.269 & 166.8 & -133.1 & -75.8 & -7.7 & -49.8 & -16.4 & -52.5 \\
\hline & p- & 2.050 & 0.799 & 0.268 & 169.6 & -134.8 & -77.1 & -7.6 & -50.5 & -17.4 & -52.4 \\
\hline \multicolumn{12}{|l|}{$\mathbf{X - P}$} \\
\hline $\mathrm{H}$ & & 2.011 & 0.749 & 0.424 & 147.3 & -153.1 & -101.7 & -7.5 & -64.2 & -19.6 & -114.3 \\
\hline \multirow[t]{3}{*}{ DMA } & o- & 2.026 & 0.750 & 0.439 & 152.8 & -157.6 & -110.8 & -12.3 & -62.4 & -17.9 & -126.9 \\
\hline & m- & 2.006 & 0.741 & 0.448 & 153.8 & -166.7 & -109.8 & -7.9 & -67.5 & -19.4 & -129.2 \\
\hline & p- & 2.002 & 0.734 & 0.462 & 155.7 & -174.0 & -111.7 & -7.7 & -68.1 & -24.2 & -136.5 \\
\hline \multirow[t]{3}{*}{$\mathrm{NH}_{2}$} & o- & 2.016 & 0.736 & 0.439 & 152.7 & -159.0 & -105.1 & -9.5 & -63.9 & -18.9 & -120.4 \\
\hline & $\mathrm{m}$ & 2.007 & 0.745 & 0.437 & 151.0 & -162.4 & -105.8 & -7.7 & -66.0 & -19.3 & -123.7 \\
\hline & $\mathrm{p}-$ & 2.005 & 0.738 & 0.450 & 152.6 & -168.4 & -107.5 & -7.6 & -66.8 & -22.3 & -130.0 \\
\hline \multirow[t]{3}{*}{$\mathrm{Br}$} & o- & 2.023 & 759 & 0.410 & 139.5 & -139.4 & -102.8 & -10.0 & -61.0 & -18.8 & -111.8 \\
\hline & m- & 2.011 & 0.750 & 0.421 & 144.0 & -143.2 & -104.6 & -7.7 & -64.5 & -19.4 & -110.1 \\
\hline & $\mathrm{p}-$ & 2.009 & 0.748 & 0.426 & 146.3 & -147.5 & -105.4 & -7.6 & -64.0 & -22.0 & -112.3 \\
\hline \multirow[t]{3}{*}{$\mathrm{SH}$} & o- & 2.017 & 0.750 & 0.419 & 148.4 & -151.0 & -105.8 & -9.7 & -63.0 & -19.3 & -117.7 \\
\hline & m- & 2.010 & 0.748 & 0.428 & 146.6 & -149.9 & -105.6 & -7.7 & -64.7 & -19.4 & -115.5 \\
\hline & p- & 2.007 & 0.744 & 0.437 & 148.5 & -153.9 & -107.6 & -7.6 & -65.1 & -23.2 & -119.1 \\
\hline \multirow[t]{3}{*}{$\mathrm{OH}$} & o & 2.020 & 0.763 & 0.411 & 140.8 & -151.5 & -99.5 & -8.6 & -60.7 & -19.0 & -118.6 \\
\hline & $\mathrm{m}-$ & 2.010 & 0.750 & 0.424 & 147.4 & -155.5 & -102.7 & -7.6 & -64.4 & -19.3 & -117.3 \\
\hline & p- & 2.009 & 0.745 & 0.434 & 148.6 & -158.0 & -103.8 & -7.6 & -65.0 & -20.8 & -119.7 \\
\hline $\mathrm{F}$ & o- & 2.023 & 0.766 & 0.395 & 133.9 & -138.2 & -95.2 & -7.9 & -58.8 & -18.8 & -106.7 \\
\hline
\end{tabular}




\begin{tabular}{|l|l|r|r|r|r|r|r|r|r|r|r|}
\hline & m- & 2.013 & 0.754 & 0.413 & 143.7 & -145.1 & -100.3 & -7.5 & -62.8 & -19.4 & -107.8 \\
\hline & p- & 2.013 & 0.751 & 0.419 & 144.9 & -147.5 & -100.9 & -7.5 & -63.3 & -19.8 & -109.6 \\
\hline $\mathrm{Cl}$ & o- & 2.024 & 0.763 & 0.403 & 137.6 & -139.2 & -100.1 & -9.4 & -60.2 & -19.1 & -110.4 \\
\hline & m- & 2.012 & 0.751 & 0.419 & 143.6 & -143.4 & -102.9 & -7.6 & -63.7 & -19.4 & -109.3 \\
\hline & p- & 2.010 & 0.749 & 0.424 & 145.7 & -147.2 & -103.8 & -7.6 & -63.6 & -21.4 & -111.5 \\
\hline $\mathrm{CH}_{3}$ & o- & 2.017 & 0.745 & 0.437 & 149.8 & -154.0 & -104.7 & -10.0 & -64.5 & -19.2 & -117.8 \\
\hline & m- & 2.009 & 0.746 & 0.432 & 148.7 & -156.1 & -104.3 & -7.7 & -65.2 & -19.7 & -118.5 \\
\hline & p- & 2.008 & 0.745 & 0.434 & 149.0 & -157.4 & -104.5 & -7.6 & -65.2 & -20.7 & -119.8 \\
\hline $\mathrm{C} \equiv \mathrm{CH}$ & o- & 2.014 & 0.764 & 0.411 & 145.1 & -150.2 & -104.7 & -10.0 & -62.2 & -20.1 & -118.7 \\
\hline & m- & 2.010 & 0.749 & 0.426 & 145.7 & -147.8 & -105.3 & -7.7 & -64.4 & -19.4 & -114.0 \\
\hline & p- & 2.007 & 0.746 & 0.432 & 147.3 & -150.2 & -107.0 & -7.6 & -64.7 & -23.7 & -116.3 \\
\hline $\mathrm{NO}_{2}$ & o- & 2.038 & 0.780 & 0.369 & 131.8 & -128.7 & -97.0 & -10.07 & -55.2 & -19.1 & -102.7 \\
\hline & m- & 2.016 & 0.757 & 0.404 & 139.4 & -132.5 & -100.1 & -7.6 & -61.4 & -19.6 & -99.3 \\
\hline & p- & 2.014 & 0.757 & 0.404 & 141.0 & -133.1 & -100.1 & -7.5 & -61.5 & -21.1 & -98.6 \\
\hline
\end{tabular}




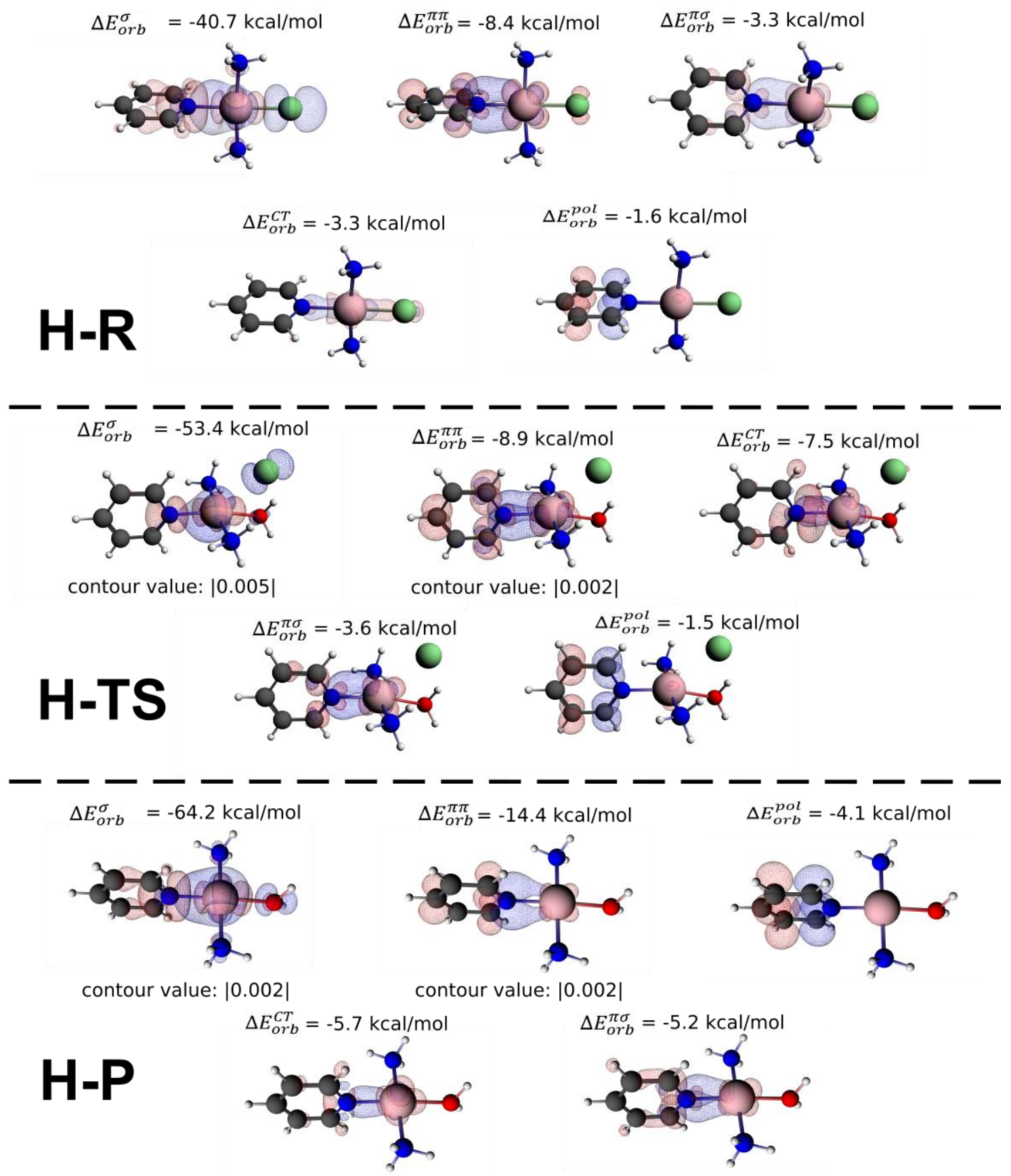

Figure S2: Five most important ETS-NOCV deformation density contributions describing the formation of the Pt-pyrX interaction in H-R, H-TS and H-P structures. $\Delta E_{\text {orb }}^{\sigma}$ represents the $\sigma$-donation, $\Delta E_{o r b}^{\pi \pi}$ and $\Delta E_{o r b}^{\pi \sigma}$ represent the $\pi$-donation with $\pi$ and $\sigma$ electrons of the pyr $\mathrm{X}$ ring involved, respectively. $\Delta E_{\text {orb }}^{C T}$ and $\Delta E_{\text {orb }}^{\text {pol }}$ represent the $\sigma$-back-donation and the pyr-X ring polarization, respectively. The blue/red contours correspond to accumulation/depletion of electron density by \pm 0.001 a.u. if not stated otherwise. 

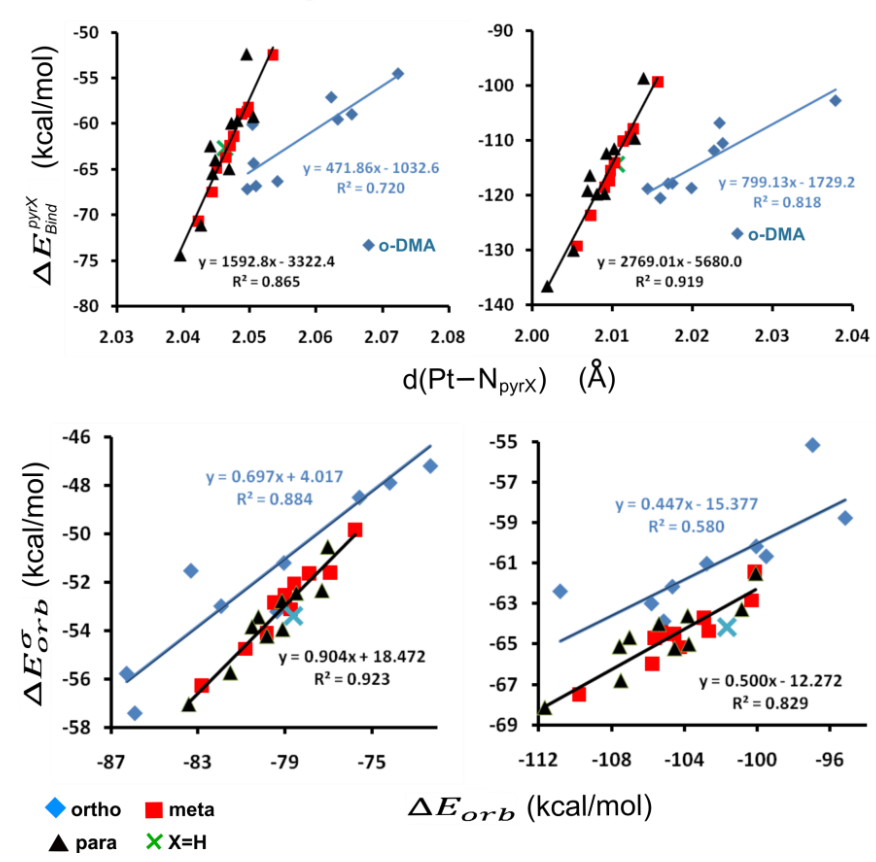

Figure S3: X-TS (left panels) and X-P (right panels) structures: Higher panels: Dependence of pyr-X ligand binding energies on the Pt- $\mathrm{N}_{\text {pyrX }}$ bond lengths. The o-DMA point was not included in the regression analysis for the ortho-X subset (blue line). Lower panels:

Dependence of the $\sigma$-donation energy $\Delta E_{\text {orb }}^{\sigma}$ contributions on total $\Delta E_{\text {orb }}$ energies. One regression line was constructed for both meta-X and para-X subsets in all graphs (black lines).

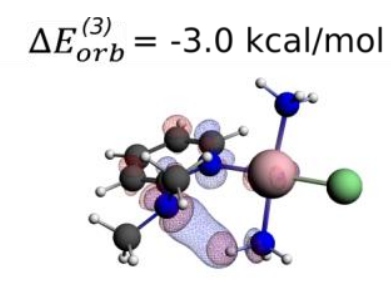

O-DMA-R

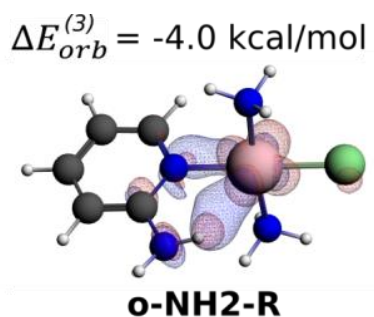

$\Delta E_{\text {orb }}^{(3)}=-4.8 \mathrm{kcal} / \mathrm{mol}$

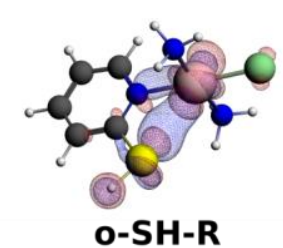

O-SH-R

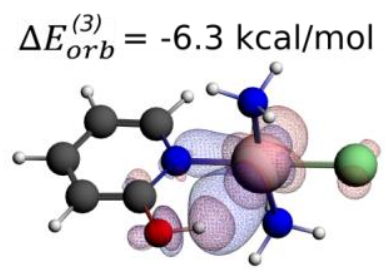

O-OH-R
$\Delta E_{\text {orb }}^{(6)}=-1.4 \mathrm{kcal} / \mathrm{mol}$

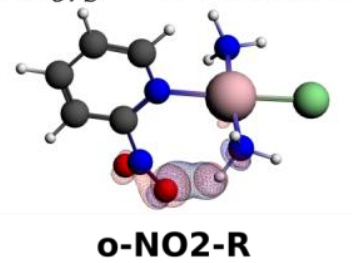

Figure S4: ETS-NOCV deformation density contributions for the formation of the Pt-pyrX interaction in X-R structures which involve contribution from $\mathrm{Pt}^{\prime \prime} \mathrm{H}\left(\mathbf{o}-\mathbf{N H}_{2}-\mathbf{R}, \mathbf{o}-\mathbf{O H}-\mathbf{R}\right)$, $\mathrm{Pt} \cdots \mathrm{S}$ (o-SH-R) nonbonding interactions or $\mathrm{NH}_{3} \cdots \mathrm{N}$ (o-DMA-R), $\mathrm{NH}_{3} \cdots \mathrm{O}\left(\mathbf{o}-\mathbf{N O}_{2}-\mathbf{R}\right) \mathrm{H}$-bond interactions. The blue/red contours correspond to accumulation/depletion of electron density by \pm 0.001 a.u. The numbers in the superscript correspond to the order of the contribution in the ETS-NOCV decomposition. 


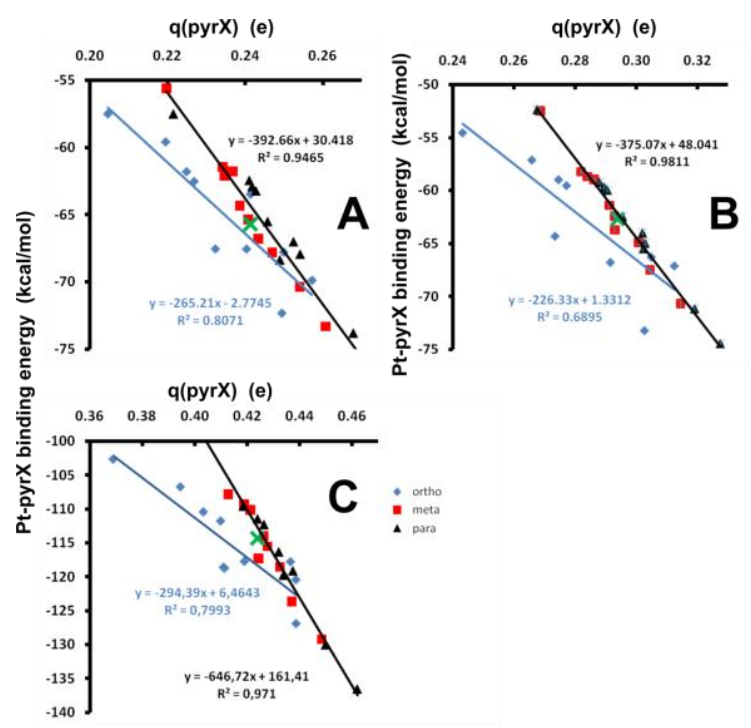

Figure S5: Dependence of the Pt-pyrX binding energies on the transferred q(pyrX) charges for X-R, X-TS and X-P structures (panels A, B and C, respectively). One regression line was constructed for meta and para substituents in all graphs (black line).
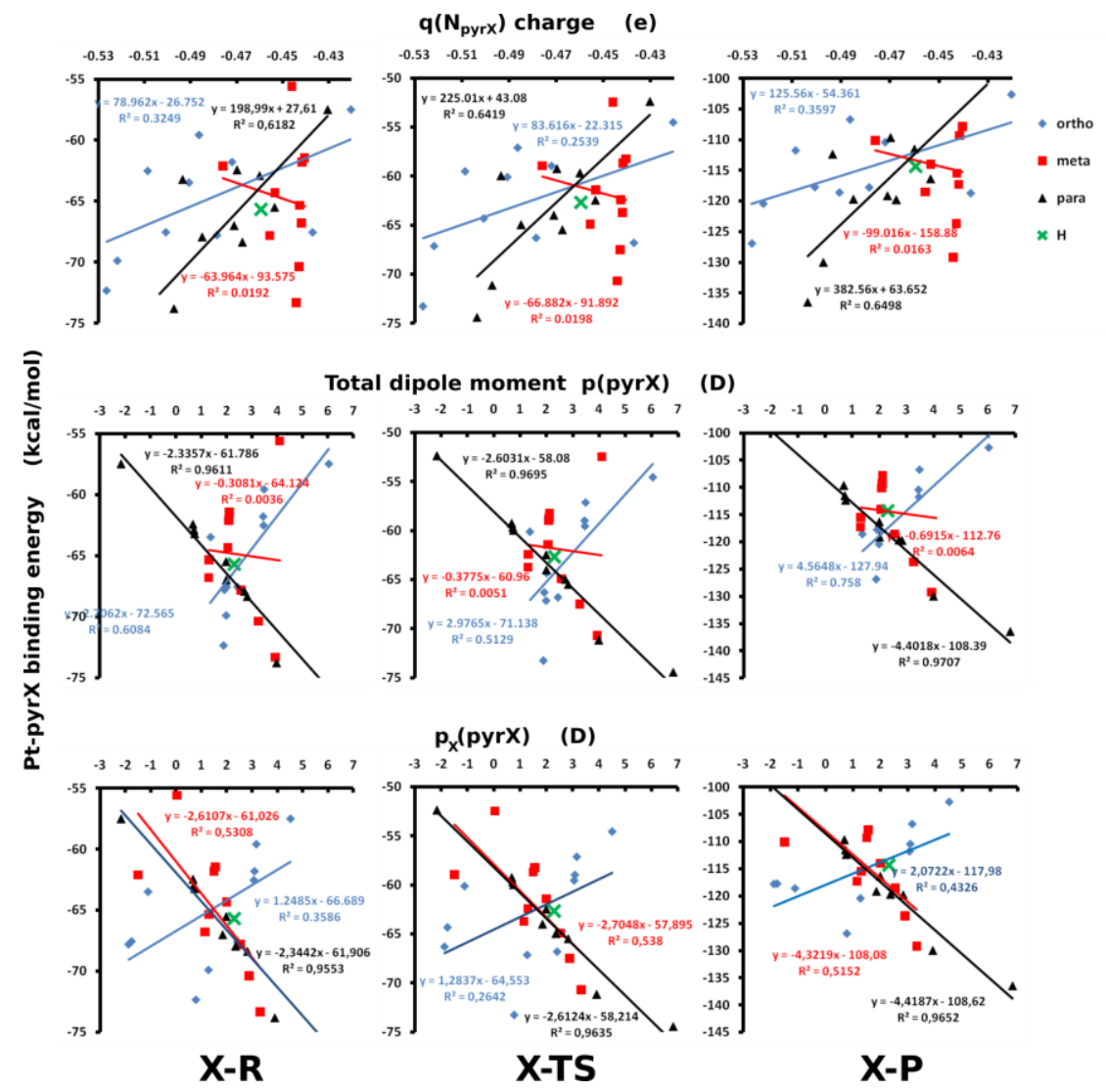

Figure S6: Dependence of the gas phase Pt-pyrX binding energies on the NBO charges of the $\mathrm{N}_{\text {pyrX }}$ atom $\left(\mathrm{q}\left(\mathrm{N}_{\mathrm{pyrX}}\right)\right)$, the total dipole moments of pyrX (p(pyrX)) and the projections of the dipole moment into $\mathrm{C} 4-\mathrm{N}_{\mathrm{pyrX}}$ direction $\left(\mathrm{p}_{\mathrm{x}}(\mathrm{pyrX})\right)$ : upper, middle and lower rows of panels, respectively for $\mathbf{X}-\mathbf{R}, \mathbf{X}-\mathbf{T S}$ and $\mathbf{X}-\mathbf{P}$ structures at the right, middle and left columns of panels, respectively. All the variables were calculated for the isolated pyrX ligands in the gas phase. 

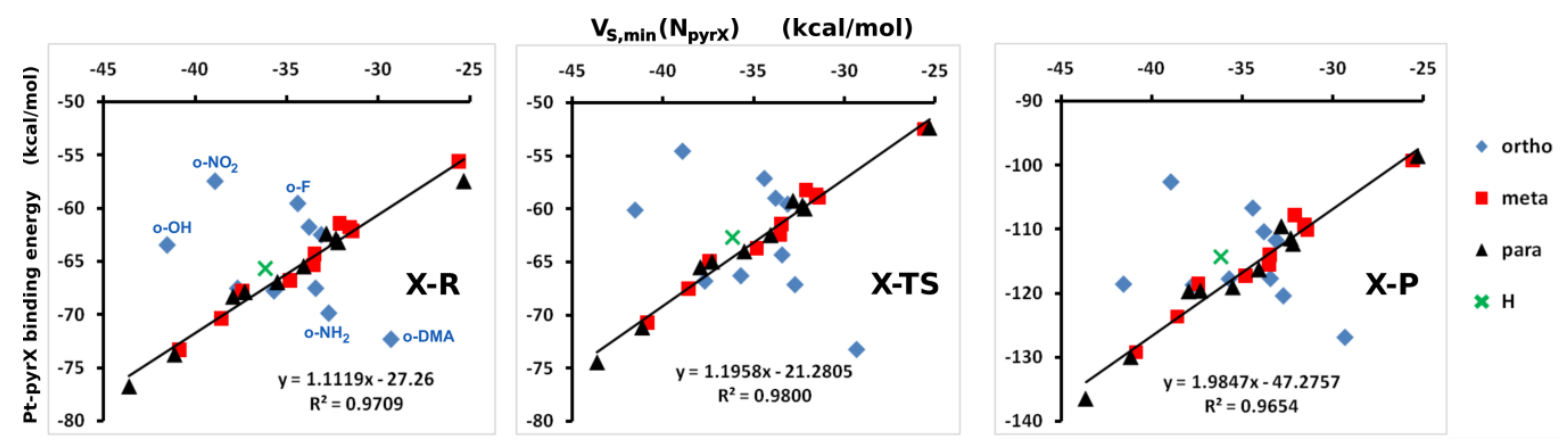

Figure S7: Dependence of the gas phase Pt-pyrX binding energies for X-R, X-TS and X-P structures on the the minimum surface electrostatic potential calculated on the surface of the $\mathrm{N}_{\mathrm{pyrX}}$ atom in the isolated pyrX ligand. One regression line was constructed for $\mathrm{m}-\mathrm{X}$ and $\mathrm{p}-\mathrm{X}$ points while excluding all o-X ones.

Table S2: Interactions of $\mathrm{Cl}$ and water ligands with the rest of the complex in $\mathbf{X}-\mathbf{R}$ and $\mathbf{X}-\mathbf{P}$ structures, respectively: $\mathrm{Pt}-\mathrm{Cl}, \mathrm{Pt}-\mathrm{O}_{\mathrm{w}}$ bond lengths (in $\AA$ ); the total NPA charges of the $\mathrm{Cl}$ and water ligands $(\mathrm{q}(\mathrm{Cl}), \mathrm{q}(\mathrm{w}))$; ETS-NOCV energy decomposition terms $\Delta E_{\text {Pauli }}, \Delta E_{\text {elst }}$, $\Delta E_{\text {orb }}, \Delta E_{\text {disp }}, \Delta E_{\text {orb }}^{\sigma}, \Delta E_{\text {orb }}^{\pi}$ were obtained at BLYP-D3BJ/TZ2P//B3LYP/BS1 level, $\Delta \mathrm{E}_{\text {Bind }}$ energies were calculated at B3LYP-D3BJ/BS2//B3LYP/BS1 level. All energy values are in $\mathrm{kcal} / \mathrm{mol}$.

\begin{tabular}{|l|l|r|r|r|r|r|r|r|r|r|}
\hline X-R & & $\mathrm{Pt}-\mathrm{Cl}$ & $\mathrm{q}(\mathrm{Cl})$ & $\Delta E_{\text {Pauli }}$ & $\Delta E_{\text {elst }}$ & $\Delta E_{\text {orb }}$ & $\Delta E_{\text {disp }}$ & $\Delta E_{\text {orb }}^{\sigma}$ & $\Delta E_{\text {orb }}^{\pi}$ & $\Delta E_{\text {bind }}$ \\
\hline $\mathrm{H}$ & & 2.315 & -0.477 & 130.5 & -283.4 & -95.3 & -3.0 & -67.5 & -9.8 & -248.7 \\
\hline DMA & $\mathrm{o}-$ & 2.316 & -0.479 & 135.1 & -277.7 & -97.9 & -3.2 & -70.1 & -10.1 & -242.4 \\
\hline & $\mathrm{m}-$ & 2.320 & -0.491 & 155.4 & -260.2 & -148.2 & -3.0 & -116.8 & -9.2 & -239.6 \\
\hline & $\mathrm{p}-$ & 2.323 & -0.498 & 128.1 & -273.4 & -89.3 & -3.0 & -64.0 & -9.9 & -235.7 \\
\hline $\mathrm{NH}_{2}$ & $\mathrm{o}-$ & 2.316 & -0.482 & 130.4 & -282.6 & -94.4 & -3.1 & -67.0 & -9.4 & -247.4 \\
\hline & $\mathrm{m}-$ & 2.317 & -0.486 & 131.8 & -278.3 & -95.8 & -3.0 & -68.5 & -9.7 & -243.6 \\
\hline & $\mathrm{p}-$ & 2.320 & -0.492 & 128.4 & -275.0 & -92.5 & -3.0 & -65.2 & -10.1 & -240.0 \\
\hline $\mathrm{Br}$ & $\mathrm{o}-$ & 2.309 & -0.467 & 132.8 & -284.7 & -96.5 & -2.9 & -67.7 & -9.7 & -249.2 \\
\hline & $\mathrm{m}-$ & 2.312 & -0.472 & 133.1 & -283.2 & -97.8 & -3.0 & -69.9 & -9.7 & -249.5 \\
\hline & $\mathrm{p}-$ & 2.314 & -0.476 & 130.9 & -282.3 & -95.9 & -3.0 & -67.5 & -10.5 & -248.6 \\
\hline $\mathrm{SH}$ & $\mathrm{o}-$ & 2.312 & -0.471 & 132.5 & -283.4 & -95.8 & -2.9 & -67.1 & -9.8 & -247.2 \\
\hline & $\mathrm{m}-$ & 2.314 & -0.477 & 130.6 & -282.3 & -94.2 & -3.0 & -67.3 & -9.6 & -246.9 \\
\hline & $\mathrm{p}-$ & 2.316 & -0.482 & 130.2 & -279.0 & -94.8 & -3.0 & -66.7 & -10.4 & -244.8 \\
\hline
\end{tabular}




\begin{tabular}{|c|c|c|c|c|c|c|c|c|c|c|}
\hline \multirow[t]{3}{*}{$\mathrm{OH}$} & - & 2.312 & -0.468 & 132.4 & -289.3 & 97.2 & -3.1 & -68.9 & -8.8 & -255.0 \\
\hline & m- & 2.315 & -0.478 & 129.0 & -280.1 & -94.6 & -3.2 & -67.7 & -9.8 & -247.1 \\
\hline & $\mathrm{p}$ & 2.316 & -0.482 & 129.5 & -280.6 & -94.1 & -3.0 & -66.5 & -10.1 & -246. \\
\hline \multirow[t]{3}{*}{$\mathrm{F}$} & o- & 2.307 & -0.464 & 132.2 & -287.9 & -97.1 & -2.7 & -68.2 & -10.0 & -252. \\
\hline & $\mathrm{n}$ & 2.311 & -0.469 & 131.4 & -286.3 & -96.6 & -3.0 & -68.6 & -10.1 & -252 . \\
\hline & p- & 2.313 & -0.472 & 130.4 & -285.7 & -95.6 & -3.0 & -67.7 & -10.1 & -251. \\
\hline \multirow[t]{3}{*}{$\mathrm{Cl}$} & 0 & 2.309 & -0.466 & 132.5 & -286.0 & -96.6 & -2.8 & -67.9 & -9.9 & -250. \\
\hline & $\mathrm{m}$ & 2.312 & -0.471 & 132.2 & -284.4 & -97.1 & -3.0 & -69.2 & -9.9 & -250 . \\
\hline & $\mathrm{p}$ & 2.313 & -0.474 & 130.8 & -283.6 & -95.8 & -3.0 & -67.7 & -10.4 & -249 . \\
\hline \multirow[t]{3}{*}{$\mathrm{CH}_{3}$} & 0 & 2.318 & -0.482 & 129.1 & -280.2 & -93.7 & -3.3 & -66.9 & -9.5 & -245 . \\
\hline & $\mathrm{m}$ & 2.316 & -0.482 & 130.7 & -280.5 & -95.0 & -3.0 & -67.3 & -9.6 & -245 . \\
\hline & $\mathrm{p}$ & 2.317 & -0.483 & 130.1 & -279.8 & -94.7 & -3.0 & -66.8 & -10.0 & -245 . \\
\hline \multirow[t]{3}{*}{$\mathrm{C} \equiv \mathrm{CH}$} & o- & 2.314 & -0.477 & 131.6 & -282.3 & -94.7 & -2.9 & -66.3 & -9.7 & -245 . \\
\hline & m- & 2.314 & -0.476 & 133.7 & -280.9 & -98.3 & -3.0 & -70.6 & -9.4 & -247.2 \\
\hline & $\mathrm{p}-$ & 2.315 & -0.479 & 130.4 & -279.8 & -95.5 & -3.0 & -66.9 & -10.5 & -245 \\
\hline \multirow[t]{3}{*}{$\mathrm{NO}_{2}$} & O- & 2.301 & -0.444 & 135.0 & -289.8 & -100.4 & -2.8 & -70.9 & -10.4 & -255 \\
\hline & m- & 2.308 & -0.460 & 135.2 & -288.7 & -100.7 & -2.9 & -72.4 & -9.9 & -256. \\
\hline & $\mathrm{p}-$ & 2.309 & -0.462 & 131.9 & -289.8 & -98.0 & -3.0 & -69.0 & -10.7 & -255.5 \\
\hline \multicolumn{2}{|l|}{ X-P } & $\mathrm{Pt}-\mathrm{O}_{\mathrm{w}}$ & $\mathrm{Pt}-\mathrm{O}_{\mathrm{w}}$ & $q(w)$ & $\Delta E_{\text {Pauli }}$ & $\Delta E_{\text {elst }}$ & $\bar{E} E_{\text {orb }}$ & $\Delta E_{\text {disp }}$ & $\Delta E_{\text {orb }}^{\sigma}$ & $\Delta E_{\text {orb }}^{\pi}$ \\
\hline $\mathrm{H}$ & & 2.137 & 0.177 & 60.9 & -64.4 & -37.9 & -3.0 & -27.9 & -5.9 & -46. \\
\hline \multirow[t]{3}{*}{ DMA } & 0 & 2.139 & 0.175 & 63.7 & -64.2 & -38.1 & -3.1 & -27.7 & -5.7 & -44 \\
\hline & $\mathrm{n}$ & 2.148 & 9 & 59.9 & -63.0 & -35.5 & -2.9 & -26.3 & -5.6 & -44. \\
\hline & $\mathrm{p}-$ & 2.152 & 0.166 & 59.1 & -61.4 & -35.2 & -2.9 & -25.9 & -5.3 & -43. \\
\hline \multirow[t]{3}{*}{$\mathrm{NH}_{2}$} & 10 & 2.141 & 0.174 & 60.1 & -63.2 & -37.0 & -3.0 & -27.3 & -5.7 & -45 . \\
\hline & $\mathrm{n}$ & 141 & 0.172 & 60.7 & -63.4 & -36.9 & -3.0 & -27.2 & -5.7 & -45 . \\
\hline & $\mathrm{p}-$ & .147 & 0.170 & 59.3 & -62.3 & -36.1 & -2.9 & -26.6 & -5.5 & -44 \\
\hline \multirow[t]{3}{*}{$\mathrm{Br}$} & 10 & 120 & 1 & 62.1 & -65.4 & -38.4 & -3.1 & -28.0 & -6.0 & -47 . \\
\hline & m- & 136 & 0.177 & 62.3 & -64.8 & -38.3 & -3.0 & -28.2 & -5.9 & -46.8 \\
\hline & p- & 38 & 0 & 60.8 & -64.1 & -37.6 & -3.0 & -27.7 & -5.8 & -46.5 \\
\hline \multirow[t]{3}{*}{$\mathrm{SH}$} & o & 2.135 & 0.176 & 61.7 & -64.7 & -37.7 & -3.1 & -27.7 & -5.9 & -46.4 \\
\hline & m- & 2.139 & 0.175 & 60.8 & -63.9 & -37.4 & -3.0 & -27.7 & -5.8 & -46.2 \\
\hline & p- & 2.142 & 0.173 & 60.2 & -63.3 & -36.9 & -3.0 & -27.2 & -5.7 & -45.4 \\
\hline
\end{tabular}




\begin{tabular}{|c|c|c|c|c|c|c|c|c|c|c|}
\hline \multirow[t]{3}{*}{$\mathrm{OH}$} & O- & 2.133 & 0.178 & 60.8 & -64.4 & -37.7 & -3.0 & -27.7 & -5.9 & -46.7 \\
\hline & m- & 2.137 & 0.176 & 61.1 & -64.3 & -37.7 & -3.0 & -27.8 & -5.9 & -46.5 \\
\hline & $\mathrm{p}-$ & 2.140 & 0.174 & 60.1 & -63.5 & -37.2 & -3.0 & -27.4 & -5.7 & -45.9 \\
\hline \multirow[t]{3}{*}{$\mathrm{F}$} & o- & 2.125 & 0.183 & 61.9 & -66.1 & -39.3 & -3.0 & -28.7 & -6.2 & -48.8 \\
\hline & m- & 2.132 & 0.179 & 61.5 & -65.2 & -38.6 & -3.0 & -28.4 & -6.1 & -47.8 \\
\hline & $\mathrm{p}-$ & 2.135 & 0.179 & 60.7 & -64.7 & -38.2 & -3.0 & -28.1 & -6.0 & -47.5 \\
\hline \multirow[t]{3}{*}{$\mathrm{Cl}$} & o- & 2.127 & 0.181 & 62.2 & -65.8 & -38.6 & -3.1 & -28.3 & -6.1 & -47.7 \\
\hline & m- & 2.136 & 0.178 & 61.7 & -64.7 & -38.3 & -3.0 & -28.3 & -6.0 & -47.1 \\
\hline & $\mathrm{p}-$ & 2.137 & 0.177 & 60.8 & -64.3 & -37.8 & -3.0 & -27.9 & -5.9 & -46.8 \\
\hline \multirow[t]{3}{*}{$\mathrm{CH}_{3}$} & o- & 2.144 & 0.172 & 60.4 & -63.3 & -36.9 & -3.0 & -27.1 & -5.7 & -45.4 \\
\hline & m- & 2.139 & 0.174 & 61.3 & -64.0 & -37.5 & -3.0 & -27.6 & -5.8 & -45.8 \\
\hline & $\mathrm{p}-$ & 2.141 & 0.174 & 60.6 & -63.6 & -37.3 & -3.0 & -27.4 & -5.7 & -45.7 \\
\hline \multirow[t]{3}{*}{$\mathrm{C} \equiv \mathrm{CH}$} & o- & 2.135 & 0.175 & 61.4 & -64.4 & -37.4 & -3.1 & -27.4 & -5.8 & -46.1 \\
\hline & m- & 2.139 & 0.175 & 62.6 & -64.5 & -38.2 & -3.0 & -28.1 & -5.8 & -46.1 \\
\hline & $\mathrm{p}-$ & 2.140 & 0.174 & 60.4 & -63.6 & -37.2 & -3.0 & -27.4 & -5.7 & -45.8 \\
\hline \multirow[t]{3}{*}{$\mathrm{NO}_{2}$} & o- & 2.116 & 0.191 & 64.1 & -67.6 & -40.7 & -3.1 & -29.7 & -6.5 & -49.7 \\
\hline & m- & 2.131 & 0.183 & 63.5 & -66.2 & -39.8 & -3.0 & -29.2 & -6.2 & -48.6 \\
\hline & p- & 2.130 & 0.182 & 61.5 & -65.6 & -39.1 & -3.0 & -28.8 & -6.2 & -48.6 \\
\hline
\end{tabular}

Table S3: The $\mathrm{Pt}-\mathrm{Cl}$ and $\mathrm{Pt}-\mathrm{O}_{\mathrm{w}}$ bonds in the gas phase optimized $\mathbf{X}$-TS structures $(\mathrm{X}=\mathrm{H}$, $\mathrm{NH}_{2}, \mathrm{NO}_{2}$ ): $\mathrm{Pt}-\mathrm{Cl}, \mathrm{Pt}-\mathrm{O}_{\mathrm{w}}$ bond lengths (in $\AA$ ); the total NPA charges of the $\mathrm{Cl}^{-}$and water fragments $(\mathrm{q}(\mathrm{Cl}), \mathrm{q}(\mathrm{w}))$ (in e).

\begin{tabular}{|l|l|l|l|l|l|}
\hline \multicolumn{2}{|l|}{} & $\mathrm{Pt}-\mathrm{Cl}$ & $\mathrm{Pt}-\mathrm{O}_{\mathrm{w}}$ & $\mathrm{q}(\mathrm{Cl})$ & $\mathrm{q}(\mathrm{w})$ \\
\hline $\mathrm{H}$ & & 2.770 & 2.327 & -0.765 & 0.058 \\
\hline DMA & o- & 2.839 & 2.294 & -0.772 & 0.058 \\
\hline & m- & 2.773 & 2.353 & -0.769 & 0.051 \\
\hline & p- & 2.782 & 2.350 & -0.770 & 0.048 \\
\hline $\mathrm{NH}_{2}$ & o- & 2.710 & 2.384 & -0.761 & 0.064 \\
\hline & m- & 2.768 & 2.344 & -0.765 & 0.054 \\
\hline & p- & 2.776 & 2.344 & -0.768 & 0.051 \\
\hline $\mathrm{Br}$ & o- & 2.778 & 2.315 & -0.762 & 0.063 \\
\hline & m- & 2.768 & 2.320 & -0.763 & 0.060 \\
\hline
\end{tabular}




\begin{tabular}{|c|c|c|c|c|c|}
\hline & $\mathrm{p}-$ & 2.764 & 2.327 & -0.764 & 0.059 \\
\hline \multirow[t]{3}{*}{$\mathrm{SH}$} & o- & 2.749 & 2.347 & -0.755 & 0.069 \\
\hline & $\mathrm{m}-$ & 2.772 & 2.326 & -0.764 & 0.058 \\
\hline & $\mathrm{p}-$ & 2.768 & 2.334 & -0.766 & 0.056 \\
\hline \multirow[t]{3}{*}{$\mathrm{OH}$} & o- & 2.691 & 2.365 & -0.764 & 0.071 \\
\hline & $\mathrm{m}-$ & 2.771 & 2.329 & -0.764 & 0.057 \\
\hline & $\mathrm{p}-$ & 2.768 & 2.333 & -0.767 & 0.056 \\
\hline \multirow[t]{3}{*}{$\mathrm{F}$} & o- & 2.775 & 2.299 & -0.764 & 0.067 \\
\hline & $\mathrm{m}-$ & 2.766 & 2.316 & -0.762 & 0.061 \\
\hline & $\mathrm{p}-$ & 2.763 & 2.321 & -0.764 & 0.061 \\
\hline \multirow[t]{3}{*}{$\mathrm{Cl}$} & O- & 2.778 & 2.310 & -0.763 & 0.064 \\
\hline & $\mathrm{m}-$ & 2.766 & 2.318 & -0.764 & 0.062 \\
\hline & p- & 2.764 & 2.325 & -0.764 & 0.060 \\
\hline \multirow[t]{3}{*}{$\mathrm{CH}_{3}$} & O- & 2.791 & 2.334 & -0.765 & 0.056 \\
\hline & $\mathrm{m}-$ & 2.771 & 2.334 & -0.766 & 0.056 \\
\hline & $\mathrm{p}-$ & 2.773 & 2.333 & -0.766 & 0.055 \\
\hline \multirow[t]{3}{*}{$\mathrm{C} \equiv \mathrm{CH}$} & O- & 2.823 & 2.322 & -0.760 & 0.053 \\
\hline & m- & 2.773 & 2.324 & -0.763 & 0.058 \\
\hline & p- & 2.770 & 2.330 & -0.765 & 0.057 \\
\hline \multirow[t]{3}{*}{$\mathrm{NO}_{2}$} & o- & 2.752 & 2.302 & -0.735 & 0.073 \\
\hline & m- & 2.760 & 2.307 & -0.758 & 0.068 \\
\hline & p- & 2.759 & 2.309 & -0.763 & 0.067 \\
\hline
\end{tabular}

Table S4: X-TS structures: ETS-NOCV energy decomposition terms $\Delta E_{\text {Pauli }}, \Delta E_{\text {elst }}, \Delta E_{\text {orb }}$, $\Delta E_{\text {disp }}, \Delta E_{\text {orb }}^{\sigma}$ for the interaction of the joint $(\mathrm{Cl}+\mathrm{w})$ fragment (leaving and entering ligands) with the rest of the complex were obtained at BLYP-D3BJ/TZ2P//B3LYP/BS1 level, $\Delta \mathrm{E}_{\text {Bind }}$ energies values of the $(\mathrm{Cl}+\mathrm{w})$ fragment and activation Gibbs energies $\Delta G^{\ddagger}$ were calculated at B3LYP-D3BJ/BS2//B3LYP/BS1 level. All energy values are in $\mathrm{kcal} / \mathrm{mol}$.

\begin{tabular}{|l|l|r|r|r|r|r|r|r|}
\hline \multicolumn{2}{|l|}{} & $\Delta E_{\text {Pauli }} \Delta E_{\text {elst }}$ & $\Delta E_{\text {orb }}$ & $\Delta E_{\text {disp }}$ & $\Delta E_{\text {orb }}^{\sigma}$ & $\Delta E_{\text {bind }}$ & $\Delta G^{\ddagger}$ \\
\hline $\mathrm{H}$ & & 88.0 & -236.2 & -69.8 & -6.0 & -37.7 & -222.9 & 33.2 \\
\hline DMA & o- & 88.0 & -227.9 & -70.2 & -7.4 & -39.3 & -217.0 & 32.9 \\
\hline
\end{tabular}




\begin{tabular}{|c|c|c|c|c|c|c|c|c|}
\hline & m- & 91.3 & -225.4 & -72.9 & -6.0 & -43.1 & -214.0 & 32.9 \\
\hline & $\mathrm{p}-$ & 85.2 & -224.1 & -63.4 & -5.9 & -35.3 & -209.8 & 33.1 \\
\hline \multirow[t]{3}{*}{$\mathrm{NH}_{2}$} & O- & 93.6 & -243.6 & -69.6 & -6.8 & -36.7 & -225.3 & 29.9 \\
\hline & m- & 87.3 & -231.2 & -68.5 & -6.0 & -37.5 & -217.9 & 32.7 \\
\hline & $\mathrm{p}-$ & 85.8 & -227.4 & -67.3 & -6.0 & -36.1 & -214.2 & 32.6 \\
\hline \multirow[t]{3}{*}{$\mathrm{Br}$} & o- & 87.7 & -235.2 & -70.0 & -6.2 & -37.7 & -222.7 & 33.7 \\
\hline & m- & 90.1 & -236.0 & -71.8 & -6.1 & -39.4 & -223.5 & 33.9 \\
\hline & p- & 88.9 & -235.4 & -70.6 & -6.1 & -37.7 & -222.8 & 33.3 \\
\hline \multirow[t]{3}{*}{$\mathrm{SH}$} & o- & 89.9 & -236.1 & -70.7 & -6.2 & -37.4 & -226.9 & 32.0 \\
\hline & m- & 89.7 & -233.3 & -71.7 & -6.1 & -40.0 & -220.9 & 33.6 \\
\hline & p- & 87.9 & -231.8 & -69.5 & -6.0 & -37.2 & -219.0 & 33.0 \\
\hline \multirow[t]{3}{*}{$\mathrm{OH}$} & O- & 98.7 & -251.3 & -73.9 & -6.8 & -38.3 & -232.4 & 30.9 \\
\hline & m- & 87.8 & -234.1 & -69.7 & -6.0 & -37.6 & -221.2 & 33.3 \\
\hline & p- & 87.2 & -233.5 & -68.8 & -6.0 & -36.9 & -220.3 & 32.9 \\
\hline \multirow[t]{3}{*}{$\mathrm{F}$} & O- & 88.1 & -239.4 & -70.9 & -6.1 & -37.9 & -226.9 & 33.6 \\
\hline & m- & 89.1 & -239.0 & -71.0 & -6.1 & -38.3 & -226.2 & 33.7 \\
\hline & p- & 88.5 & -238.9 & -70.3 & -6.1 & -37.6 & -225.8 & 33.3 \\
\hline \multirow[t]{3}{*}{$\mathrm{Cl}$} & o- & 87.9 & -236.8 & -70.1 & -6.2 & -37.8 & -224.2 & 33.7 \\
\hline & m- & 89.7 & -237.3 & -71.6 & -6.1 & -39.0 & -224.6 & 33.9 \\
\hline & $\mathrm{p}-$ & 88.8 & -236.7 & -70.5 & -6.1 & -37.7 & -223.8 & 33.3 \\
\hline \multirow[t]{3}{*}{$\mathrm{CH}_{3}$} & O- & 87.4 & -234.0 & -68.8 & -6.2 & -37.0 & -220.9 & 32.8 \\
\hline & m- & 87.9 & -233.2 & -69.3 & -6.0 & -37.6 & -220.0 & 32.7 \\
\hline & $\mathrm{p}-$ & 87.5 & -232.3 & -69.3 & -6.0 & -37.3 & -219.3 & 33.0 \\
\hline \multirow[t]{3}{*}{$\mathrm{C} \equiv \mathrm{CH}$} & O- & 86.7 & -232.3 & -68.8 & -6.1 & -36.9 & -219.7 & 33.3 \\
\hline & m- & 89.9 & -233.2 & -71.9 & -6.0 & -40.1 & -221.2 & 34.5 \\
\hline & p- & 88.1 & -232.5 & -70.2 & -6.0 & -37.3 & -220.1 & 33.1 \\
\hline \multirow[t]{3}{*}{$\mathrm{NO}_{2}$} & o- & 91.4 & -242.2 & -74.0 & -6.1 & -39.8 & -230.0 & 33.3 \\
\hline & m- & 92.2 & -241.8 & -74.6 & -6.1 & -41.5 & -230.4 & 34.4 \\
\hline & p- & 90.8 & -243.2 & -73.2 & -6.1 & -39.4 & -230.8 & 34.3 \\
\hline
\end{tabular}




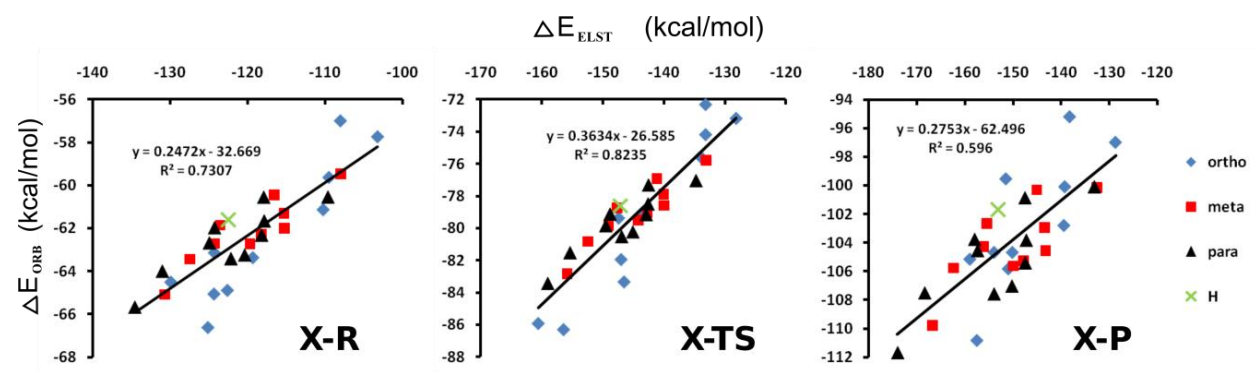

Figure S8: Correlation between $\Delta E_{\text {elst }}$ and $\Delta E_{\text {orb }}$ terms for the Pt-pyrX interaction in $\mathbf{X}-\mathbf{R}$, X-TS and X-P structures (left, center and right panels, respectively)

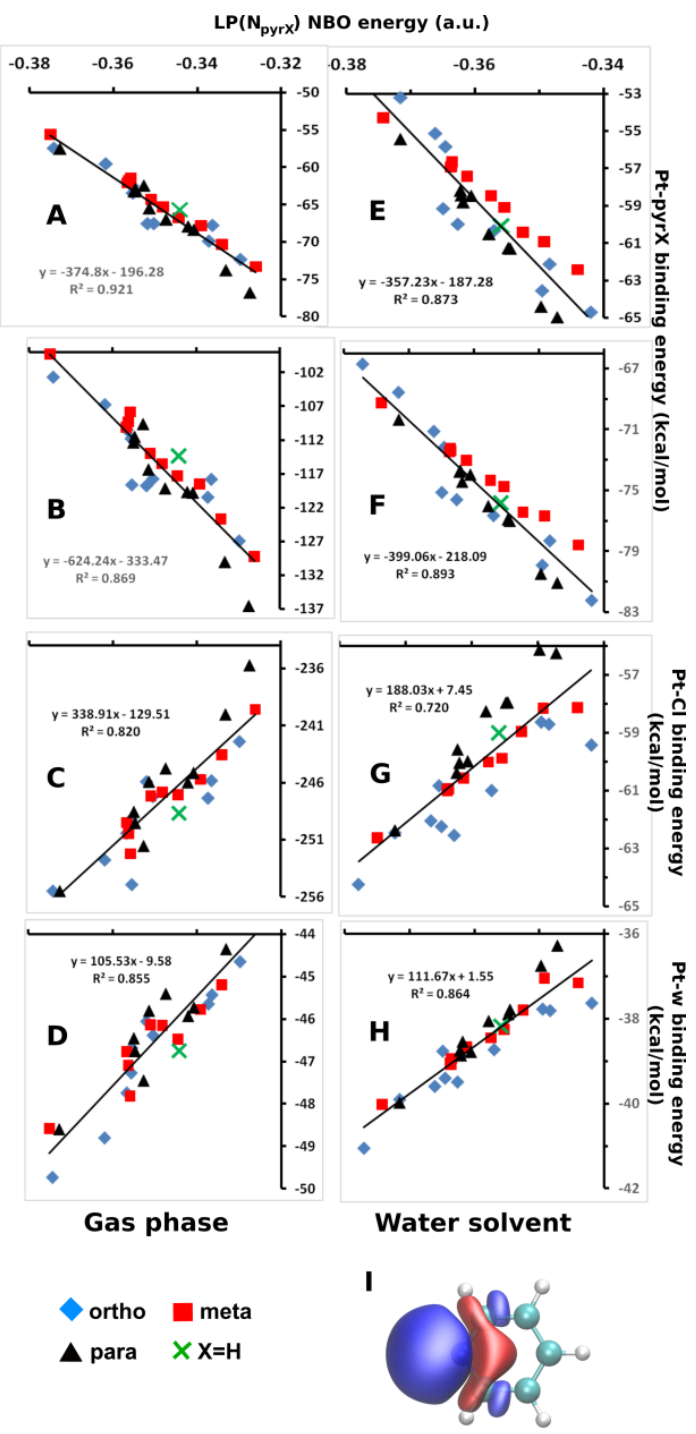

Figure S9: Dependence of the $\mathrm{Pt}-\operatorname{pyr}(\mathrm{X})$ (panels A, B), $\mathrm{Pt}-\mathrm{Cl}$ (panel C) and $\mathrm{Pt}-\mathrm{O}_{\mathrm{w}}$ (panel D) gas phase binding energies in $\mathbf{X}-\mathbf{R}$ and $\mathbf{X}-\mathbf{P}$ complexes (A, C and B, D panels, respectively) on the $\mathrm{LP}\left(\mathrm{N}_{\mathrm{pyrX}}\right) \mathrm{NBO}$ energies calculated on the isolated pyrX ligands. Panels $\mathrm{E}, \mathrm{F}, \mathrm{G}, \mathrm{H}$ represent analogous results calculated in the water solvent. $\mathrm{LP}\left(\mathrm{N}_{\mathrm{pyrX}}\right) \mathrm{NBO}$ for the pyrH ligand is shown in the panel I. 
Table S5: Bonding interactions in X-R and X-P structures optimized in the water phase and calculated by the B3LYP-D3BJ-PCM/BS2//B3LYP-PCM/BS1 method: $\mathrm{Pt}-\mathrm{N}_{\text {pyrX }}, \mathrm{Pt}-\mathrm{Cl}$ and $\mathrm{Pt}-\mathrm{O}_{\mathrm{w}}$ bond lengths (in $\AA$ ); the total NPA charges of the pyrX, $\mathrm{Cl}$ and water ligands (q(pyrX), $\mathrm{q}(\mathrm{Cl})$ and $\mathrm{q}(\mathrm{w})$, respectively) (in e); $\Delta E_{\text {Bind }}$ energy values in $\mathrm{kcal} / \mathrm{mol}$.

\begin{tabular}{|c|c|c|c|c|c|c|c|}
\hline \multicolumn{2}{|l|}{ X-R } & \multirow{2}{*}{\begin{tabular}{|r} 
Pt- $\mathrm{N}_{\text {pyrX }}$ \\
2.052
\end{tabular}} & \multirow{2}{*}{$\begin{array}{r}\mathrm{q}(\mathrm{pyrX}) \\
0.284\end{array}$} & \multirow{2}{*}{$\begin{array}{r}\Delta E_{\text {bind }}^{\text {pyrX }} \\
-43.9\end{array}$} & \multirow{2}{*}{$\begin{array}{c}\text { Pt-Cl } \\
2.370\end{array}$} & \multirow{2}{*}{$\begin{array}{l}\mathrm{q}(\mathrm{Cl}) \\
-0.597\end{array}$} & \multirow{2}{*}{$\frac{\Delta E_{\text {bind }}^{C l}}{-38.7}$} \\
\hline $\mathrm{H}$ & & & & & & & \\
\hline \multirow{3}{*}{\begin{tabular}{|l} 
DMA \\
\end{tabular}} & O- & 2.074 & 0.283 & -46.2 & 2.360 & -0.588 & -38.5 \\
\hline & m- & 2.053 & 0.295 & -46.3 & 2.368 & -0.602 & -37.7 \\
\hline & p- & 2.047 & 0.310 & -48.3 & 2.377 & -0.612 & -36.9 \\
\hline \multirow[t]{3}{*}{$\mathrm{NH}_{2}$} & o- & 2.064 & 0.292 & -46.2 & 2.363 & -0.594 & -38.2 \\
\hline & m- & 2.051 & 0.289 & -45.3 & 2.363 & -0.599 & -38.5 \\
\hline & p- & 2.047 & 0.312 & -47.7 & 2.378 & -0.610 & -37.0 \\
\hline \multirow[t]{3}{*}{$\mathrm{Br}$} & o- & 2.079 & 0.256 & -39.4 & 2.348 & -0.574 & -40.8 \\
\hline & m- & 2.059 & 0.268 & -41.4 & 2.359 & -0.587 & -39.4 \\
\hline & p- & 2.056 & 0.274 & -42.3 & 2.365 & -0.592 & -39.5 \\
\hline \multirow[t]{3}{*}{$\mathrm{SH}$} & o- & 2.064 & 0.264 & -43.6 & 2.354 & -0.583 & -39.6 \\
\hline & m- & 2.059 & 0.276 & -43.0 & 2.356 & -0.590 & -39.2 \\
\hline & p- & 2.052 & 0.288 & -44.2 & 2.370 & -0.599 & -38.4 \\
\hline \multirow[t]{3}{*}{$\mathrm{OH}$} & o- & 2.064 & 0.263 & -42.8 & 2.355 & -0.576 & -40.4 \\
\hline & $\mathrm{m}-$ & 2.055 & 0.280 & -43.4 & 2.358 & -0.593 & -38.5 \\
\hline & p- & 2.053 & 0.293 & -44.9 & 2.371 & -0.601 & -38.2 \\
\hline \multirow[t]{3}{*}{$\mathrm{F}$} & o- & 2.074 & 0.256 & -37.5 & 2.350 & -0.577 & -40.9 \\
\hline & m- & 2.059 & 0.267 & -41.5 & 2.356 & -0.586 & -39.4 \\
\hline & p- & 2.056 & 0.277 & -42.4 & 2.367 & -0.592 & -39.4 \\
\hline \multirow[t]{3}{*}{$\mathrm{Cl}$} & o- & 2.074 & 0.256 & -39.1 & 2.350 & -0.577 & -40.6 \\
\hline & m- & 2.060 & 0.267 & -41.4 & 2.353 & -0.585 & -39.8 \\
\hline & p- & 2.053 & 0.275 & -42.3 & 2.368 & -0.592 & -39.2 \\
\hline \multirow[t]{3}{*}{$\mathrm{CH}_{3}$} & o- & 2.059 & 0.286 & -45.3 & 2.369 & -0.597 & -38.4 \\
\hline & m- & 2.053 & 0.287 & -44.7 & 2.361 & -0.597 & -38.6 \\
\hline & p- & 2.049 & 0.291 & -44.9 & 2.373 & -0.601 & -38.1 \\
\hline \multirow[t]{3}{*}{$\mathrm{C} \equiv \mathrm{CH}$} & O- & 2.061 & 0.274 & -42.7 & 2.358 & -0.586 & -39.7 \\
\hline & m- & 2.060 & 0.270 & -42.1 & 2.354 & -0.587 & -39.6 \\
\hline & p- & 2.053 & 0.275 & -42.9 & 2.366 & -0.593 & -39.3 \\
\hline \multirow[t]{3}{*}{$\mathrm{NO}_{2}$} & o- & 2.084 & 0.231 & -35.4 & 2.341 & -0.560 & -42.3 \\
\hline & m- & 2.067 & 0.251 & -38.8 & 2.353 & -0.576 & -41.2 \\
\hline & p- & 2.056 & 0.247 & -39.7 & 2.360 & -0.580 & -40.8 \\
\hline X-P & & $\mathrm{Pt}-\mathrm{N}_{\mathrm{pyrX}}$ & q(pyrX) & $\Delta E_{\text {bind }}^{\text {pyrX }}$ & $\mathrm{Pt}-\mathrm{O}_{\mathrm{w}}$ & $q(w)$ & $\Delta E_{\text {bind }}^{W}$ \\
\hline $\mathrm{H}$ & & 2.011 & 0.356 & -54.3 & 2.118 & 0.195 & -20.5 \\
\hline DMA & O- & 2.028 & 0.360 & -57.0 & 2.118 & 0.197 & -19.7 \\
\hline
\end{tabular}




\begin{tabular}{|c|c|c|c|c|c|c|c|}
\hline & m- & 2.008 & 0.370 & -57.5 & 2.125 & 0.191 & -19.9 \\
\hline & p- & 2.005 & 0.388 & -60.2 & 2.132 & 0.187 & -19.2 \\
\hline \multirow[t]{3}{*}{$\mathrm{NH}_{2}$} & o- & 2.021 & 0.371 & -56.7 & 2.123 & 0.195 & -19.7 \\
\hline & m- & 2.008 & 0.364 & -55.9 & 2.120 & 0.194 & -19.9 \\
\hline & p- & 2.010 & 0.383 & -58.9 & 2.123 & 0.189 & -19.7 \\
\hline \multirow[t]{3}{*}{$\mathrm{Br}$} & o- & 2.027 & 0.338 & -49.4 & 2.118 & 0.202 & -21.1 \\
\hline & m- & 2.014 & 0.343 & -51.5 & 2.119 & 0.198 & -20.9 \\
\hline & p- & 2.012 & 0.347 & -52.4 & 2.114 & 0.198 & -20.8 \\
\hline \multirow[t]{3}{*}{$\mathrm{SH}$} & O- & 2.017 & 0.347 & -53.7 & 2.123 & 0.198 & -20.2 \\
\hline & m- & 2.015 & 0.351 & -53.4 & 2.118 & 0.196 & -20.0 \\
\hline & p- & 2.012 & 0.360 & -54.8 & 2.117 & 0.194 & -20.3 \\
\hline \multirow[t]{3}{*}{$\mathrm{OH}$} & o- & 2.021 & 0.343 & -52.4 & 2.122 & 0.200 & -20.2 \\
\hline & m- & 2.011 & 0.353 & -53.9 & 2.116 & 0.196 & -19.9 \\
\hline & p- & 2.013 & 0.365 & -55.5 & 2.118 & 0.193 & -20.2 \\
\hline \multirow[t]{3}{*}{$\mathrm{F}$} & o- & 2.028 & 0.328 & -47.2 & 2.108 & 0.204 & -21.1 \\
\hline & m- & 2.013 & 0.342 & -51.4 & 2.119 & 0.197 & -20.4 \\
\hline & p- & 2.014 & 0.349 & -52.6 & 2.114 & 0.198 & -20.8 \\
\hline \multirow[t]{3}{*}{$\mathrm{Cl}$} & o- & 2.024 & 0.336 & -49.0 & 2.114 & 0.201 & -20.8 \\
\hline & m- & 2.015 & 0.343 & -51.4 & 2.120 & 0.198 & -21.2 \\
\hline & p- & 2.014 & 0.347 & -52.5 & 2.113 & 0.197 & -20.7 \\
\hline \multirow[t]{3}{*}{$\mathrm{CH}_{3}$} & O- & 2.022 & 0.362 & -55.9 & 2.125 & 0.193 & -19.8 \\
\hline & m- & 2.008 & 0.361 & -55.5 & 2.121 & 0.194 & -20.2 \\
\hline & p- & 2.010 & 0.363 & -55.5 & 2.118 & 0.194 & -20.3 \\
\hline \multirow[t]{3}{*}{$\mathrm{C} \equiv \mathrm{CH}$} & o- & 2.015 & 0.349 & -52.9 & 2.118 & 0.198 & -20.7 \\
\hline & m- & 2.015 & 0.346 & -52.4 & 2.122 & 0.196 & -20.5 \\
\hline & p- & 2.010 & 0.348 & -53.1 & 2.115 & 0.197 & -20.7 \\
\hline \multirow[t]{3}{*}{$\mathrm{NO}_{2}$} & o- & 2.034 & 0.306 & -44.2 & 2.098 & 0.211 & -21.7 \\
\hline & m- & 2.014 & 0.327 & -48.5 & 2.114 & 0.202 & -21.0 \\
\hline & p- & 2.011 & 0.322 & -49.1 & 2.108 & 0.203 & -21.4 \\
\hline
\end{tabular}




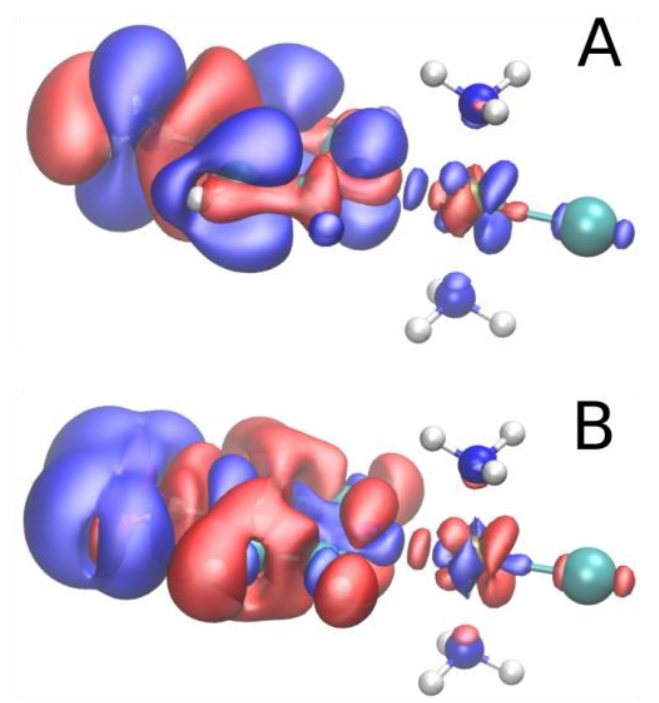

Figure S10: Electron density difference isosurfaces of $\mathbf{p}-\mathbf{N H}_{\mathbf{2}}-\mathbf{R}$ (A) and $\mathbf{p}-\mathbf{N O}_{\mathbf{2}}-\mathbf{R}$ (B) structures with respect to the reference H-R structure calculated in the water solvent. They show electron accumulation (blue: 0.0004 a.u.) and depletion (red: -0.0004 a.u.) regions caused by $\mathrm{p}-\mathrm{NH}_{2}$ (A) and $\mathrm{p}-\mathrm{NO}_{2}$ (B) substitution of the pyr ring. Electron densities were calculated on the H-R geometry for all atoms of respective complexes except the $\mathrm{X}$ substituent atoms whose positions were optimized.

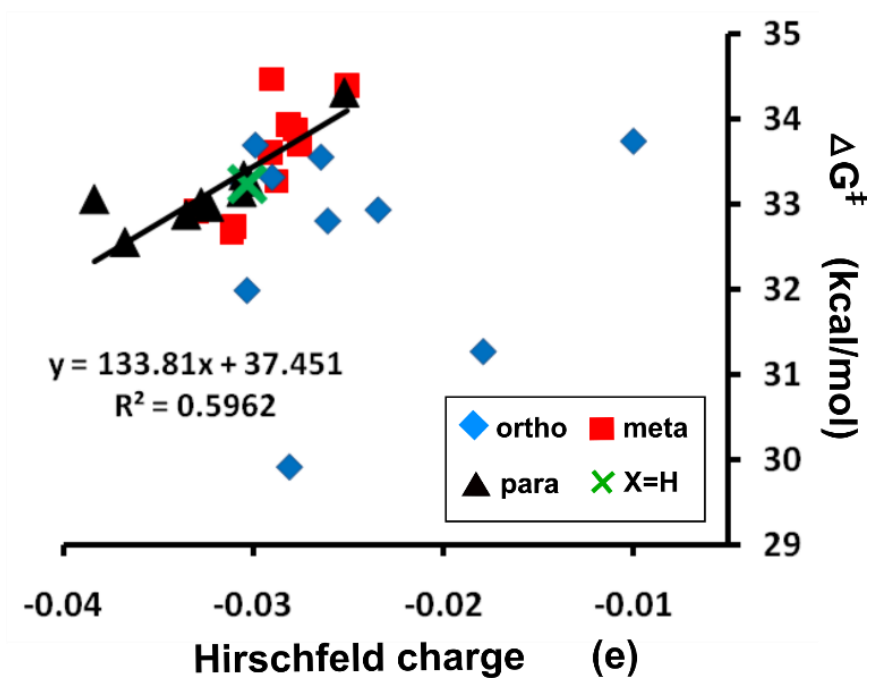

Figure S11: Dependence of the gas phase activation Gibbs free energies $\left(\Delta \mathrm{G}^{*}\right)$ on the Hirschfeld charges calculated on the Pt(II) atom. One regression line was constructed for $\mathrm{m}-\mathrm{X}$ and $\mathrm{p}-\mathrm{X}$ reaction paths while excluding all $\mathrm{o}-\mathrm{X}$ points. 
Table S6: Activation free energies $\left(\Delta G^{\ddagger}\right)$ and bonding interactions in X-TS structures optimized in the water solvent and calculated by the B3LYP-D3BJ-PCM/BS2//B3LYPPCM/BS1 method: $\mathrm{Pt}-\mathrm{N}_{\text {pyrX }}, \mathrm{Pt}-\mathrm{Cl}$ and $\mathrm{Pt}-\mathrm{O}_{\mathrm{w}}$ bond lengths (in $\AA$ ); the total NPA charges of the pyrX, $\mathrm{Cl}$ and water ligands $(\mathrm{q}(\mathrm{pyrX}), \mathrm{q}(\mathrm{Cl})$ and $\mathrm{q}(\mathrm{w})$, respectively) (in e); all energy values are in $\mathrm{kcal} / \mathrm{mol}$.

\begin{tabular}{|c|c|c|c|c|c|c|c|c|c|c|}
\hline \multicolumn{2}{|l|}{ X-TS } & \multirow{2}{*}{$\begin{array}{r}\mathrm{Pt}-\mathrm{N}_{\text {pyrX }} \\
2.034\end{array}$} & \multirow{2}{*}{$\begin{array}{r}q(\text { pyrX }) \\
0.318\end{array}$} & \multirow{2}{*}{\begin{tabular}{|r|}
$\Delta E_{\text {bind }}^{\text {pyrX }}$ \\
-42.9 \\
\end{tabular}} & \multirow{2}{*}{\begin{tabular}{|l|}
$\mathrm{Pt}-\mathrm{Cl}$ \\
2.837 \\
\end{tabular}} & \multirow{2}{*}{$\begin{array}{c}\mathrm{q}(\mathrm{Cl}) \\
-0.837\end{array}$} & \multirow{2}{*}{\begin{tabular}{|l|}
$\mathrm{Pt}-\mathrm{O}_{\mathrm{w}}$ \\
2.476
\end{tabular}} & \multirow{2}{*}{$\begin{array}{l}q(w) \\
0.050\end{array}$} & \multirow{2}{*}{$\begin{array}{r}\Delta E_{\text {bind }}^{(x+C l)} \\
-18.6\end{array}$} & \multirow{2}{*}{\begin{tabular}{|l}
$\Delta G^{\ddagger}$ \\
25.9
\end{tabular}} \\
\hline $\mathrm{H}$ & & & & & & & & & & \\
\hline \multirow[t]{3}{*}{ DMA } & o- & 2.056 & 0.318 & -45.8 & 2.880 & -0.841 & 2.430 & 0.058 & -18.8 & 26.7 \\
\hline & $\mathrm{m}$ & 2.032 & 0.334 & -45.3 & 2.842 & -0.838 & 2.486 & 0.048 & -17.4 & 26.5 \\
\hline & p- & 2.025 & 0.353 & -47.5 & 2.846 & -0.841 & 2.508 & 0.042 & -16.7 & 26.3 \\
\hline \multirow[t]{3}{*}{$\mathrm{NH}_{2}$} & 0 & 2.042 & 0.333 & -45.0 & 2.806 & -0.832 & 2.482 & 0.053 & -18.5 & 24.3 \\
\hline & $\mathrm{m}$ & 2.032 & 0.327 & -44.3 & 2.843 & -0.841 & 2.469 & 0.050 & -18.1 & 26.0 \\
\hline & p- & 2.029 & 350 & -47.0 & 2.846 & -0.841 & 2.495 & 0.044 & -17.3 & 25.4 \\
\hline \multirow[t]{3}{*}{$\mathrm{Br}$} & o- & 2.051 & 0.292 & -37.8 & 2.816 & -0.828 & 2.462 & 0.058 & -20.4 & 26.2 \\
\hline & $\mathrm{n}$ & 2.037 & 0.305 & -40.0 & 2.835 & -0.837 & 2.457 & 0.056 & -19.7 & 27.0 \\
\hline & $\mathrm{p}$ & 2.035 & 0.306 & -40.9 & 2.821 & -0.833 & 2.471 & 0.051 & -19.1 & 26.1 \\
\hline \multirow[t]{3}{*}{$\mathrm{SH}$} & O- & 2.049 & 0.295 & -42.4 & 2.797 & -0.829 & 2.448 & 0.061 & -20.2 & 25.9 \\
\hline & $\mathrm{n}$ & 2.032 & 0.313 & -42.0 & 2.840 & -0.839 & 2.458 & 0.055 & -19.1 & 26.3 \\
\hline & p- & 2.032 & 0.322 & -42.9 & 2.827 & -0.835 & 2.482 & 0.049 & -18.4 & 25.9 \\
\hline \multirow[t]{3}{*}{$\mathrm{OH}$} & 0 & 2.045 & .296 & -41.3 & 2.761 & -0.818 & 2.477 & 0.060 & -20.9 & 25.7 \\
\hline & m- & 2.033 & 315 & -42.6 & 2.841 & -0.840 & 2.457 & 0.053 & -18.3 & 26.2 \\
\hline & p- & 2.034 & 0.328 & -43.8 & 2.834 & -0.837 & 2.482 & 0.048 & -18.3 & 25.7 \\
\hline \multirow[t]{3}{*}{$\mathrm{F}$} & o- & 2.054 & 280 & 36.1 & 2.815 & -0.831 & 2.437 & 0.061 & -21.3 & 25.9 \\
\hline & m- & 2.037 & 298 & 9.9 & 2.819 & -1 & 2.454 & 0 & 9.5 & 26.4 \\
\hline & $\mathrm{p}$ & 2.038 & 308 & 1.1 & 2.823 & -0.833 & 2.471 & 0.052 & -19.1 & 26.1 \\
\hline \multirow[t]{3}{*}{$\mathrm{Cl}$} & o- & 2.061 & 0.285 & -37.7 & 2.816 & -0.833 & 2.428 & 0.064 & -20.6 & 26.3 \\
\hline & m- & 2.034 & 0.301 & -40.3 & 2.834 & -0.837 & 2.450 & 0.057 & -19.7 & 26.5 \\
\hline & p- & 2.035 & 0.307 & -41.0 & 2.830 & -0.835 & 2.467 & 0.053 & -19.5 & 26.3 \\
\hline \multirow[t]{2}{*}{$\mathrm{CH}_{3}$} & O- & 2.041 & 0.326 & -44.6 & 2.840 & -0.837 & 2.477 & 0.052 & -19.3 & 25.5 \\
\hline & $\mathrm{m}-$ & 2.035 & 0.321 & -43.1 & 2.826 & -0.839 & 2.464 & 0.052 & -18.0 & 25.8 \\
\hline
\end{tabular}




\begin{tabular}{|l|l|r|r|r|r|r|r|r|r|l|}
\hline & p- & 2.031 & 0.326 & -43.8 & 2.837 & -0.836 & 2.486 & 0.048 & -18.5 & 25.8 \\
\hline $\mathrm{C} \equiv \mathrm{CH}$ & o- & 2.041 & 0.297 & -41.5 & 2.811 & -0.831 & 2.453 & 0.059 & -20.0 & 26.2 \\
\hline & m- & 2.033 & 0.306 & -41.0 & 2.837 & -0.838 & 2.455 & 0.056 & -19.1 & 27.2 \\
\hline & p- & 2.033 & 0.307 & -41.5 & 2.823 & -0.833 & 2.475 & 0.051 & -18.9 & 26.0 \\
\hline $\mathrm{NO}_{2}$ & o- & 2.074 & 0.252 & -33.0 & 2.783 & -0.821 & 2.431 & 0.067 & -21.9 & 26.5 \\
\hline & m- & 2.042 & 0.281 & -37.2 & 2.822 & -0.830 & 2.447 & 0.059 & -20.2 & 27.3 \\
\hline & p- & 2.035 & 0.272 & -38.1 & 2.816 & -0.828 & 2.448 & 0.059 & -19.6 & 27.2 \\
\hline
\end{tabular}

Table S7: Complexes with poly-F ligands: calculated $X_{c}$ and estimated $X_{e}$ values of binding energies (in $\mathrm{kcal} / \mathrm{mol}$ ), ligand charges (in e), bond lengths of $\mathbf{X}-\mathbf{R}$ structures for polysubstituted complexes together with activation free energies of the respective pathways. Relative and absolute errors are also shown. Estimated values were obtained from Equation 2; relative errors from the ratio $\frac{\left|X_{C}-X_{e}\right| * 100 \%}{\left|X_{e}-X_{H}\right|}$, where $X_{H}$ are the calculated values for the H-R complex; absolute errors represent the difference $\left|X_{c}-X_{e}\right|$.

\begin{tabular}{|l|r|r|r|r|r|r|r|}
\hline & \multicolumn{1}{|c|}{$\Delta E_{\text {bind }}^{\text {pyr }}$} & \multicolumn{1}{|c|}{$\Delta E_{\text {bind }}^{C l}$} & $\mathrm{q}(\mathrm{Cl})$ & $\mathrm{q}(\mathrm{pyrX})$ & \multicolumn{1}{l}{$\mathrm{Pt}-\mathrm{N}_{\text {pyrX }}$} & \multicolumn{1}{l|}{$\mathrm{Pt}-\mathrm{Cl}$} & \multicolumn{1}{|c|}{$\Delta G^{*}$} \\
\hline Gas phase & & & & & & & \\
\hline 2m-F & -57.3 & -255.9 & -0.461 & 0.224 & 2.088 & 2.308 & 34.4 \\
\hline om-F & -55.6 & -256.1 & -0.457 & 0.214 & 2.103 & 2.305 & 33.9 \\
\hline op-F & -56.5 & -255.5 & -0.459 & 0.221 & 2.104 & 2.305 & 33.5 \\
\hline o2mp-F & -49.0 & -261.7 & -0.445 & 0.204 & 2.112 & 2.300 & 34.0 \\
\hline 2op-F & -41.5 & -258.8 & -0.445 & 0.195 & 2.126 & 2.300 & 35.1 \\
\hline 2o2mp-F & -264.9 & -0.431 & 0.182 & 2.135 & 2.295 & 35.5 \\
\hline 2m-F (estimated) & -57.2 & -255.8 & -0.461 & 0.227 & 2.088 & 2.308 & 34.2 \\
\hline om-F (estimated) & -55.3 & -256.4 & -0.456 & 0.212 & 2.104 & 2.304 & 33.8 \\
\hline op-F (estimated) & -56.3 & -255.7 & -0.459 & 0.219 & 2.104 & 2.305 & 33.7 \\
\hline o2mp-F (estimated) & -47.8 & -262.8 & -0.443 & 0.205 & 2.111 & 2.298 & 34.6 \\
\hline 2op-F (estimated) & -50.2 & -259.8 & -0.445 & 0.197 & 2.123 & 2.298 & 33.8 \\
\hline 2o2mp-F & & & & & & & \\
(estimated) & -41.7 & -266.9 & -0.429 & 0.183 & 2.131 & 2.291 & 34.7 \\
\hline 2m-F (rel. err.) & 1.5 & 0.8 & 0.5 & 22.8 & 0.4 & 4.4 & 21.9 \\
\hline om-F (rel. err.) & 3.0 & 2.9 & 3.7 & 6.2 & 1.2 & 6.7 & 11.0 \\
\hline op-F (rel. err.) & 1.8 & 2.8 & 2.3 & 6.1 & 0.2 & 3.3 & 42.7 \\
\hline o2mp-F (rel. err.) & 6.5 & 8.1 & 6.6 & 3.3 & 1.3 & 11.5 & 41.9 \\
\hline 2op-F (rel. err.) & 8.6 & 8.7 & 0.8 & 5.8 & 6.6 & 10.7 & 242.7 \\
\hline 2o2mp-F (rel. err.) & 10.5 & 11.1 & 3.7 & 2.5 & 8.3 & 17.0 & 51.5 \\
\hline 2m-F (abs. err.) & 0.1 & 0.1 & 0.000 & 0.003 & 0.000 & 0.000 & 0.2 \\
\hline om-F (abs. err.) & 0.3 & 0.2 & 0.001 & 0.002 & 0.000 & 0.001 & 0.1 \\
\hline op-F (abs. err.) & 0.2 & 0.2 & 0.000 & 0.001 & 0.000 & 0.000 & 0.2 \\
\hline o2mp-F (abs. err.) & 1.2 & 1.1 & 0.002 & 0.001 & 0.000 & 0.002 & 0.6 \\
\hline
\end{tabular}




\begin{tabular}{|l|r|r|r|r|r|r|r|}
\hline 2op-F (abs. err.) & 1.3 & 1.0 & 0.000 & 0.003 & 0.003 & 0.002 & 1.3 \\
\hline 2o2mp-F (abs. err.) & 2.5 & 2.0 & 0.002 & 0.001 & 0.004 & 0.004 & 0.8 \\
\hline \hline Water solvent & & & & & & & \\
\hline 2m-F & -39.0 & -40.7 & -0.579 & 0.254 & 2.059 & 2.358 & 27.1 \\
\hline om-F & -35.2 & -41.8 & -0.569 & 0.242 & 2.078 & 2.343 & 26.1 \\
\hline op-F & -36.1 & -40.9 & -0.574 & 0.251 & 2.077 & 2.351 & 25.9 \\
\hline o2mp-F & -31.5 & -43.1 & -0.559 & 0.228 & 2.086 & 2.345 & 26.9 \\
\hline 2op-F & -30.6 & -42.6 & -0.559 & 0.229 & 2.114 & 2.342 & 27.5 \\
\hline 2o2mp-F & -26.5 & -44.6 & -0.545 & 0.207 & 2.108 & 2.338 & 27.0 \\
\hline 2m-F (estimated) & -39.1 & -40.0 & -0.576 & 0.251 & 2.066 & 2.342 & 27.0 \\
\hline om-F (estimated) & -35.1 & -41.5 & -0.566 & 0.239 & 2.080 & 2.336 & 26.5 \\
\hline op-F (estimated) & -36.0 & -41.6 & -0.572 & 0.249 & 2.078 & 2.347 & 26.1 \\
\hline o2mp-F (estimated) & -31.2 & -42.9 & -0.551 & 0.216 & 2.091 & 2.319 & 27.3 \\
\hline 2op-F (estimated) & -29.6 & -43.7 & -0.552 & 0.221 & 2.099 & 2.328 & 26.2 \\
\hline 2o2mp-F & & & & & & & \\
(estimated) & -24.8 & -45.0 & -0.531 & 0.189 & 2.112 & 2.300 & 27.3 \\
\hline 2m-F (rel. err.) & 2.7 & 53.6 & -18.0 & 10.0 & 46.9 & 58.3 & 7.7 \\
\hline om-F (rel. err.) & 0.6 & 9.3 & 8.9 & 5.9 & 6.5 & 20.2 & 6.4 \\
\hline op-F (rel. err.) & 1.0 & 22.9 & 8.9 & 4.3 & 3.6 & 15.0 & 75.6 \\
\hline o2mp-F (rel. err.) & 2.3 & 6.6 & 17.4 & 17.8 & 12.4 & 51.1 & 5.6 \\
\hline 2op-F (rel. err.) & 7.1 & 22.4 & 16.3 & 11.8 & 32.8 & 33.0 & 453.6 \\
\hline 2o2mp-F (rel. err.) & 9.0 & -6.7 & 21.3 & 19.8 & 7.6 & 54.8 & 23.0 \\
\hline 2m-F (abs. err.) & 0.1 & 0.7 & 0.004 & 0.003 & 0.006 & 0.016 & 0.1 \\
\hline om-F (abs. err.) & 0.1 & 0.3 & 0.003 & 0.003 & 0.002 & 0.007 & 0.4 \\
\hline op-F (abs. err.) & 0.1 & 0.7 & 0.002 & 0.001 & 0.001 & 0.003 & 0.2 \\
\hline o2mp-F (abs. err.) & 0.3 & 0.3 & 0.008 & 0.012 & 0.005 & .026 & 0.4 \\
\hline 2op-F (abs. err.) & 1.0 & .1 & 0.007 & 0.007 & 0.015 & 0.014 & 1.4 \\
\hline 2o2mp-F (abs. err.) & 1.7 & -0.4 & 0.014 & 0.019 & 0.005 & 0.038 & 0.3 \\
\hline
\end{tabular}

Table S8: Complexes with poly-F ligands: calculated $X_{c}$ and estimated $X_{e}$ values of binding energies (in $\mathrm{kcal} / \mathrm{mol}$ ), ligand charges (in e), bond lengths of $\mathbf{X}-\mathbf{P}$ structures for polysubstituted complexes. Relative and absolute errors are also shown. Estimated values were obtained from Equation 2; relative errors from the ratio $\frac{\left|X_{c}-X_{e}\right| * 100 \%}{\left|X_{e}-X_{H}\right|}$, where $X_{H}$ are the values for the H-R complex; absolute errors represent the difference $\left|X_{c}-X_{e}\right|$.

\begin{tabular}{|l|r|r|r|r|r|r|}
\hline & \multicolumn{1}{|c|}{$\Delta \mathrm{E}_{\text {bind }}^{\text {pyrX }}$} & $\Delta \mathrm{E}_{\text {bind }}^{\mathrm{w}}$ & $\mathrm{q}(\mathrm{pyrX})$ & $\mathrm{q}(\mathrm{w})$ & Pt-N $_{\text {pyrX }}$ & $\mathrm{Pt}^{-\mathrm{O}_{\mathrm{w}}}$ \\
\hline Gas phase & & & & & & \\
\hline $2 \mathrm{~m}-\mathrm{F}$ & -101.4 & -48.9 & 0.401 & 0.184 & 2.015 & 2.128 \\
\hline om-F & -100.5 & -49.8 & 0.385 & 0.186 & 2.026 & 2.119 \\
\hline op-F & -102.3 & -49.3 & 0.391 & 0.186 & 2.025 & 2.124 \\
\hline o2mp-F & -90.3 & -51.2 & 0.373 & 0.191 & 2.032 & 2.115 \\
\hline 2op-F & -95.7 & -51.1 & 0.363 & 0.192 & 2.041 & 2.112 \\
\hline 2o2mp-F & -83.7 & -52.6 & 0.348 & 0.197 & 2.047 & 2.107 \\
\hline
\end{tabular}




\begin{tabular}{|c|c|c|c|c|c|c|}
\hline 2m-F (estimated) & -101.4 & -48.9 & 0.402 & 0.182 & 2.015 & 2.126 \\
\hline om-F (estimated) & -100.3 & -49.9 & 0.384 & 0.186 & 2.025 & 2.119 \\
\hline op-F (estimated) & -102.0 & -49.5 & 0.389 & 0.186 & 2.026 & 2.122 \\
\hline o2mp-F (estimated) & -89.1 & -51.6 & 0.367 & 0.191 & 2.030 & 2.111 \\
\hline 2op-F (estimated) & -94.4 & -51.6 & 0.360 & 0.192 & 2.038 & 2.110 \\
\hline 2o2mp-F (estimated) & -81.5 & -53.7 & 0.338 & 0.198 & 2.042 & 2.098 \\
\hline 2m-F (rel. err.) & 0.4 & 0.2 & 2.2 & 29.4 & 17.7 & 18.0 \\
\hline om-F (rel. err.) & 1.8 & 3.5 & 3.1 & 2.8 & 3.9 & 1.4 \\
\hline op-F (rel. err.) & 2.3 & 7.2 & 4.8 & 1.1 & 2.8 & 12.6 \\
\hline o2mp-F (rel. err.) & 4.8 & 10.0 & 10.4 & 0.9 & 10.5 & 14.6 \\
\hline 2op-F (rel. err.) & 6.2 & 10.3 & 5.0 & 3.2 & 8.5 & 9.6 \\
\hline 2o2mp-F (rel. err.) & 6.8 & 15.6 & 11.8 & 3.8 & 13.0 & 21.0 \\
\hline $2 \mathrm{~m}-\mathrm{F}$ (abs. err.) & 0.1 & 0.0 & 0.000 & 0.002 & 0.001 & 0.002 \\
\hline om-F (abs. err.) & 0.2 & 0.1 & 0.001 & 0.000 & 0.001 & 0.000 \\
\hline op-F (abs. err.) & 0.3 & 0.2 & 0.002 & 0.000 & 0.000 & 0.002 \\
\hline o2mp-F (abs. err.) & 1.2 & 0.5 & 0.006 & 0.000 & 0.002 & 0.004 \\
\hline 2op-F (abs. err.) & 1.2 & 0.5 & 0.003 & 0.001 & 0.002 & 0.003 \\
\hline 2o2mp-F (abs. err.) & 2.2 & 1.1 & 0.010 & 0.001 & 0.004 & 0.008 \\
\hline \multicolumn{7}{|l|}{ Water solvent } \\
\hline $2 \mathrm{~m}-\mathrm{F}$ & -48.6 & -21.2 & 0.328 & 0.203 & 2.014 & 2.112 \\
\hline om-F & -44.3 & -21.58 & 0.315 & 0.208 & 2.030 & 2.099 \\
\hline op-F & -45.3 & -20.6 & 0.324 & 0.204 & 2.029 & 2.110 \\
\hline o $2 \mathrm{mp}-\mathrm{F}$ & -39.9 & -22.3 & 0.299 & 0.211 & 2.031 & 2.104 \\
\hline 2op-F & -39.3 & -22.1 & 0.299 & 0.209 & 2.044 & 2.103 \\
\hline 2o2mp-F & -34.0 & -22.7 & 0.276 & 0.217 & 2.050 & 2.098 \\
\hline $2 \mathrm{~m}-\mathrm{F}$ (estimated) & -48.5 & -20.3 & 0.327 & 0.200 & 2.015 & 2.120 \\
\hline om-F (estimated) & -44.2 & -21.0 & 0.314 & 0.206 & 2.029 & 2.109 \\
\hline op-F (estimated) & -45.4 & -21.4 & 0.321 & 0.206 & 2.031 & 2.105 \\
\hline o2mp-F (estimated) & -39.6 & -21.3 & 0.292 & 0.210 & 2.034 & 2.107 \\
\hline 2op-F (estimated) & -38.2 & -22.0 & 0.293 & 0.214 & 2.047 & 2.096 \\
\hline 2o2mp-F (estimated) & -32.4 & -21.9 & 0.264 & 0.219 & 2.051 & 2.098 \\
\hline $2 \mathrm{~m}-\mathrm{F}$ (rel. err.) & 3.0 & 652.8 & 2.5 & 77.6 & 25.9 & 363.4 \\
\hline om-F (rel. err.) & 0.7 & 113.4 & 1.8 & 22.1 & 3.3 & 126.4 \\
\hline op-F (rel. err.) & 1.7 & 90.1 & 10.3 & 24.5 & 7.1 & 39.0 \\
\hline o2mp-F (rel. err.) & 2.3 & 120.1 & 11.8 & 3.9 & 12.3 & 30.8 \\
\hline 2op-F (rel. err.) & 6.4 & 5.7 & 9.5 & 27.8 & 8.9 & 35.2 \\
\hline 2o2mp-F (rel. err & 7.4 & 58.1 & 13.1 & 8.7 & 2.1 & 3.0 \\
\hline 2m-F (abs. err.) & 0.2 & 0.9 & 0.001 & 0.003 & 0.001 & 0.008 \\
\hline om-F (abs. err.) & 0.1 & 0.6 & 0.001 & 0.002 & 0.001 & 0.010 \\
\hline op-F (abs. err.) & 0.1 & 0.9 & 0.004 & 0.003 & 0.001 & 0.005 \\
\hline o2mp-F (abs. err.) & 0.3 & 1.0 & 0.008 & 0.001 & 0.003 & 0.003 \\
\hline 2op-F (abs. err.) & 1.0 & 0.1 & 0.006 & 0.005 & 0.003 & 0.008 \\
\hline 2o2mp-F (abs. err.) & 1.6 & 0.8 & 0.012 & 0.002 & 0.001 & 0.001 \\
\hline
\end{tabular}


Table S9: Complexes with poly- $\mathbf{N H}_{2}$ ligands: calculated $X_{c}$ and estimated $X_{e}$ values of binding energies (in $\mathrm{kcal} / \mathrm{mol}$ ), ligand charges (in e), bond lengths of $\mathbf{X}-\mathbf{R}$ structures for polysubstituted complexes together with activation free energies of the respective pathways. Relative and absolute errors are also shown. Estimated values were obtained from Equation 2; relative errors from the ratio $\frac{\left|X_{C}-X_{e}\right| * 100 \%}{\left|X_{e}-X_{H}\right|}$, where $X_{H}$ are the calculated values for the H-R complex; absolute errors represent the difference $\left|X_{c}-X_{e}\right|$.

\begin{tabular}{|c|c|c|c|c|c|c|c|}
\hline & $\Delta E_{\text {bind }}^{\text {pyrX }}$ & $\Delta E_{\text {bind }}^{C l}$ & $\mathrm{q}(\mathrm{Cl})$ & $q(p y r X)$ & Pt-N ${ }_{\text {pyrX }}$ & $\mathrm{Pt}-\mathrm{Cl}$ & $\Delta G^{\ddagger}$ \\
\hline \multicolumn{8}{|l|}{ Gas phase } \\
\hline $2 \mathrm{~m}-\mathrm{NH}_{2}$ & -74.1 & -239.8 & -0.492 & 0.260 & 2.076 & 2.320 & 33.4 \\
\hline om- $\mathrm{NH}_{2}$ & -73.8 & -243.0 & -0.487 & 0.262 & 2.085 & 2.317 & 30.9 \\
\hline op- $\mathrm{NH}_{2}$ & -77.3 & -239.6 & -0.493 & 0.273 & 2.083 & 2.320 & 30.8 \\
\hline 2op- $\mathrm{NH}_{2}$ & -81.0 & -239.2 & -0.491 & 0.284 & 2.097 & 2.317 & 30.5 \\
\hline $2 \mathrm{~m}-\mathrm{NH}_{2}$ (estimated) & -75.0 & -238.4 & -0.494 & 0.267 & 2.076 & 2.320 & 32.1 \\
\hline om- $\mathrm{NH}_{2}$ (estimated) & -74.6 & -242.2 & -0.490 & 0.270 & 2.084 & 2.319 & 29.3 \\
\hline op- $\mathrm{NH}_{2}$ (estimated) & -78.0 & -238.7 & -0.497 & 0.284 & 2.083 & 2.322 & 29.2 \\
\hline 2op- $\mathrm{NH}_{2}$ (estimated) & -82.2 & -237.4 & -0.501 & 0.300 & 2.088 & 2.323 & 25.9 \\
\hline $2 \mathrm{~m}-\mathrm{NH}_{2}$ (rel. err.) & 9.9 & 13.0 & 12.8 & 24.6 & 5.7 & 8.5 & 113.5 \\
\hline om- $\mathrm{NH}_{2}$ (rel. err.) & 8.4 & 11.2 & 22.4 & 27.4 & 39.5 & 51.7 & 40.0 \\
\hline op- $\mathrm{NH}_{2}$ (rel. err.) & 6.0 & 9.0 & 17.5 & 24.2 & 30.3 & 21.7 & 38.6 \\
\hline 2op- $\mathrm{NH}_{2}$ (rel. err.) & 7.3 & 16.1 & 41.9 & 27.1 & 115.8 & 74.5 & 62.0 \\
\hline $2 \mathrm{~m}-\mathrm{NH}_{2}$ (abs. err.) & 0.9 & 1.3 & 0.002 & 0.006 & 0.000 & 0.000 & 1.3 \\
\hline om- $\mathrm{NH}_{2}$ (abs. err.) & 0.7 & 0.7 & 0.003 & 0.008 & 0.001 & 0.002 & 1.6 \\
\hline op- $\mathrm{NH}_{2}$ (abs. err.) & 0.7 & 0.9 & 0.003 & 0.010 & 0.001 & 0.001 & 1.5 \\
\hline 2op- $\mathrm{NH}_{2}$ (abs. err.) & 1.2 & 1.8 & 0.010 & 0.016 & 0.009 & 0.006 & 4.5 \\
\hline \multicolumn{8}{|l|}{ Water solvent } \\
\hline $2 \mathrm{~m}-\mathrm{NH}_{2}$ & -45.8 & -37.8 & -0.603 & 0.296 & 2.052 & 2.370 & 26.5 \\
\hline om- $\mathrm{NH}_{2}$ & -46.3 & -38.1 & -0.596 & 0.295 & 2.064 & 2.362 & 25.5 \\
\hline op- $\mathrm{NH}_{2}$ & -48.11 & -37.4 & -0.604 & 0.310 & 2.060 & 2.368 & 25.7 \\
\hline 2op- $\mathrm{NH}_{2}$ & -48.7 & -38.0 & -0.597 & 0.312 & 2.074 & 2.375 & 24.5 \\
\hline $2 \mathrm{~m}-\mathrm{NH}_{2}$ (estimated) & -46.7 & -38.3 & -0.6 & 0.295 & 2.050 & 2.356 & 26.2 \\
\hline om- $\mathrm{NH}_{2}$ (estimated) & -47.6 & -38.0 & -0.6 & 0.298 & 2.062 & 2.356 & 24.5 \\
\hline op- $\mathrm{NH}_{2}$ (estimated) & -50.0 & -36.4 & -0.6 & 0.320 & 2.058 & 2.372 & 23.8 \\
\hline 2op- $\mathrm{NH}_{2}$ (estimated) & -52.3 & -35.9 & -0.606 & 0.328 & 2.069 & 2.365 & 22.3 \\
\hline $2 \mathrm{~m}-\mathrm{NH}_{2}$ (rel. err.) & 33.2 & 100.8 & 21.9 & 7.6 & 95.0 & 100.2 & 110.9 \\
\hline om- $\mathrm{NH}_{2}$ (rel. err.) & 34.6 & 24.1 & 211.8 & 20.5 & 21.4 & 43.8 & 74.7 \\
\hline op- $\mathrm{NH}_{2}$ (rel. err.) & 31.2 & 42.9 & 38.0 & 27.0 & 34.8 & 199.5 & 91.3 \\
\hline 2op- $\mathrm{NH}_{2}$ (rel. err.) & 42.9 & 74.7 & 97.3 & 36.8 & 30.1 & 209.7 & 63.3 \\
\hline $2 \mathrm{~m}-\mathrm{NH}_{2}$ (abs. err.) & 0.9 & 0.4 & 0.001 & 0.001 & 0.003 & 0.014 & 0.4 \\
\hline om- $\mathrm{NH}_{2}$ (abs. err.) & 1.3 & 0.2 & 0.001 & 0.003 & 0.002 & 0.006 & 1.0 \\
\hline op- $\mathrm{NH}_{2}$ (abs. err.) & 1.9 & 1.0 & 0.004 & 0.010 & 0.002 & 0.004 & 1.9 \\
\hline 2op- $\mathrm{NH}_{2}$ (abs. err.) & 3.6 & 2.1 & 0.009 & 0.016 & 0.005 & 0.009 & 2.3 \\
\hline
\end{tabular}


Table S10: Complexes with poly- $\mathbf{N H}_{2}$ ligands: calculated $X_{c}$ and estimated $X_{e}$ values of binding energies (in $\mathrm{kcal} / \mathrm{mol}$ ), ligand charges (in e), bond lengths of $\mathbf{X}-\mathbf{P}$ structures for polysubstituted complexes. Relative and absolute errors are also shown. Estimated values were obtained from Equation 2; relative errors from the ratio $\frac{\left|X_{C}-X_{e}\right| * 100 \%}{\left|X_{e}-X_{H}\right|}$, where $X_{H}$ are the values for the H-R complex; absolute errors represent the difference $\left|X_{C}-X_{e}\right|$.

\begin{tabular}{|c|c|c|c|c|c|c|}
\hline & $\Delta E_{\text {bind }}^{\text {pyrX }}$ & $\Delta E_{\text {bind }}^{W}$ & $q(p y r X)$ & $q(w)$ & Pt-N $\mathrm{N}_{\text {pyrX }}$ & $\mathrm{Pt}-\mathrm{O}_{\mathrm{w}}$ \\
\hline \multicolumn{7}{|l|}{ Gas phase } \\
\hline $2 \mathrm{~m}-\mathrm{NH}_{2}$ & -131.4 & -44.1 & 0.447 & 0.170 & 2.005 & 2.146 \\
\hline om- $\mathrm{NH}_{2}$ & -130.7 & -44.4 & 0.446 & 0.172 & 2.015 & 2.141 \\
\hline op- $\mathrm{NH}_{2}$ & -135.5 & -43.7 & 0.458 & 0.169 & 2.013 & 2.146 \\
\hline 2op- $\mathrm{NH}_{2}$ & -142.2 & -43.3 & 0.475 & 0.170 & 2.028 & 2.144 \\
\hline $2 \mathrm{~m}-\mathrm{NH}_{2}$ (estimated) & -133.0 & -43.6 & 0.450 & 0.168 & 2.004 & 2.145 \\
\hline om- $\mathrm{NH}_{2}$ (estimated) & -129.8 & -44.1 & 0.452 & 0.170 & 2.013 & 2.145 \\
\hline op- $\mathrm{NH}_{2}$ (estimated) & -136.1 & -43.3 & 0.465 & 0.167 & 2.011 & 2.150 \\
\hline 2op- $\mathrm{NH}_{2}$ (estimated) & -142.2 & -42.2 & 0.479 & 0.164 & 2.016 & 2.154 \\
\hline $2 \mathrm{~m}-\mathrm{NH}_{2}$ (rel. err.) & 8.9 & 14.6 & 12.4 & 19.2 & 13.7 & 9.3 \\
\hline om- $\mathrm{NH}_{2}$ (rel. err.) & 5.7 & 10.4 & 20.3 & 29.3 & 125.0 & 55.4 \\
\hline op- $\mathrm{NH}_{2}$ (rel. err.) & 2.8 & 11.9 & 15.8 & 19.9 & 4551.8 & 32.4 \\
\hline 2op- $\mathrm{NH}_{2}$ (rel. err.) & 0.2 & 25.2 & 7.4 & 48.9 & 223.7 & 61.2 \\
\hline $2 \mathrm{~m}-\mathrm{NH}_{2}$ (abs. err.) & 1.7 & 0.5 & 0.003 & 0.002 & 0.001 & 0.001 \\
\hline om- $\mathrm{NH}_{2}$ (abs. err.) & 0.9 & 0.3 & 0.006 & 0.002 & 0.003 & 0.004 \\
\hline op- $\mathrm{NH}_{2}$ (abs. err.) & 0.6 & 0.4 & 0.006 & 0.002 & 0.003 & 0.004 \\
\hline 2op- $\mathrm{NH}_{2}$ (abs. err.) & 0.1 & 1.2 & 0.004 & 0.006 & 0.012 & 0.010 \\
\hline \multicolumn{7}{|l|}{ Water solvent } \\
\hline $2 \mathrm{~m}-\mathrm{NH}_{2}$ & -56.7 & -19.4 & 0.370 & 0.191 & 2.009 & 2.124 \\
\hline om- $\mathrm{NH}_{2}$ & -57.2 & -19.4 & 0.372 & 0.193 & 2.019 & 2.121 \\
\hline op- $\mathrm{NH}_{2}$ & -56.7 & -19.3 & 0.387 & 0.189 & 2.017 & 2.129 \\
\hline 2op- $\mathrm{NH}_{2}$ & -59.6 & -19.7 & 0.397 & 0.192 & 2.034 & 2.126 \\
\hline $2 \mathrm{~m}-\mathrm{NH}_{2}$ (estimated) & -57.4 & -19.4 & 0.372 & 0.192 & 2.006 & 2.122 \\
\hline om- $\mathrm{NH}_{2}$ (estimated) & -58.3 & -19.2 & 0.379 & 0.193 & 2.018 & 2.125 \\
\hline op- $\mathrm{NH}_{2}$ (estimated) & -61.3 & -18.9 & 0.398 & 0.188 & 2.020 & 2.128 \\
\hline 2op- $\mathrm{NH}_{2}$ (estimated) & 63.7 & 18.2 & 0.412 & 0.187 & 2.030 & 2.134 \\
\hline $2 \mathrm{~m}-\mathrm{NH}_{2}$ (rel. err.) & 22.8 & 4.4 & 10.3 & 12.4 & 56.8 & 53.9 \\
\hline om- $\mathrm{NH}_{2}$ (rel. err.) & 26.4 & 20.7 & 27.8 & 14.5 & 11.7 & 51.8 \\
\hline op- $\mathrm{NH}_{2}$ (rel. err.) & 65.6 & 28.0 & 25.3 & 22.9 & 37.8 & 4.7 \\
\hline 2op- $\mathrm{NH}_{2}$ (rel. err.) & 43.9 & 64.9 & 27.1 & 56.1 & 24.7 & 46.8 \\
\hline 2m- $\mathrm{NH}_{2}$ (abs. err.) & 0.7 & 0.0 & 0.002 & 0.000 & 0.003 & 0.002 \\
\hline om- $\mathrm{NH}_{2}$ (abs. err.) & 1.1 & 0.3 & 0.006 & 0.000 & 0.001 & 0.004 \\
\hline op- $\mathrm{NH}_{2}$ (abs. err.) & 4.6 & 0.4 & 0.011 & 0.002 & 0.003 & 0.001 \\
\hline 2op- $\mathrm{NH}_{2}$ (abs. err.) & 4.1 & 1.5 & 0.015 & 0.005 & 0.005 & 0.008 \\
\hline
\end{tabular}


Table S11: Complexes with poly-NO $\mathbf{N O}_{2}$ ligands: calculated $X_{c}$ and estimated $X_{e}$ values of binding energies (in $\mathrm{kcal} / \mathrm{mol}$ ), ligand charges (in e), bond lengths of $\mathbf{X}-\mathbf{R}$ structures for polysubstituted complexes together with activation free energies of the respective pathways. Relative and absolute errors are also shown. Estimated values were obtained from Equation 2; relative errors from the ratio $\frac{\left|X_{c}-X_{e}\right| * 100 \%}{\left|X_{e}-X_{H}\right|}$, where $X_{H}$ are the calculated values for the H-R complex; absolute errors represent the difference $\left|X_{c}-X_{e}\right|$.

\begin{tabular}{|c|c|c|c|c|c|c|c|}
\hline & $\Delta E_{\text {bind }}^{\text {pyrX }}$ & $\Delta E_{\text {bind }}^{C l}$ & $\mathrm{q}(\mathrm{Cl})$ & $q(p y r X)$ & Pt-N ${ }_{\text {pyrX }}$ & $\mathrm{Pt}-\mathrm{Cl}$ & $\Delta G^{*}$ \\
\hline \multicolumn{8}{|l|}{ Gas phase } \\
\hline $2 \mathrm{~m}-\mathrm{NO}_{2}$ & -46.9 & -262.6 & -0.446 & 0.201 & 2.096 & 2.302 & 35.1 \\
\hline om- $\mathrm{NO}_{2}$ & -49.1 & -262.1 & -0.429 & 0.188 & 2.115 & 2.296 & 34.9 \\
\hline op- $\mathrm{NO}_{2}$ & -49.2 & -262.7 & -0.431 & 0.185 & 2.111 & 2.297 & 34.7 \\
\hline $2 \mathrm{~m}-\mathrm{NO}_{2}$ (estimated) & -45.5 & -263.3 & -0.444 & 0.198 & 2.096 & 2.301 & 35.6 \\
\hline om- $\mathrm{NO}_{2}$ (estimated) & -47.4 & -262.8 & -0.427 & 0.183 & 2.119 & 2.295 & 34.5 \\
\hline op- $\mathrm{NO}_{2}$ (estimated) & -49.3 & -262.4 & -0.429 & 0.185 & 2.114 & 2.296 & 34.4 \\
\hline $2 \mathrm{~m}-\mathrm{NO}_{2}$ (rel. err.) & 7.0 & 5.1 & 6.6 & 5.7 & 5.8 & 6.4 & 19.2 \\
\hline om- $\mathrm{NO}_{2}$ (rel. err.) & 9.3 & 5.0 & 4.3 & 8.3 & 10.7 & 9.4 & 35.6 \\
\hline op- $\mathrm{NO}_{2}$ (rel. err.) & 0.7 & 2.1 & 3.8 & 0.4 & 9.9 & 7.5 & 20.9 \\
\hline $2 \mathrm{~m}-\mathrm{NO}_{2}$ (abs. err.) & 1.4 & 0.8 & 0.002 & 0.002 & 0.001 & 0.001 & 0.4 \\
\hline om- $\mathrm{NO}_{2}$ (abs. err.) & 1.7 & 0.7 & 0.002 & 0.005 & 0.004 & 0.002 & 0.4 \\
\hline op- $\mathrm{NO}_{2}$ (abs. err.) & 0.1 & 0.3 & 0.002 & 0.000 & 0.003 & 0.001 & 0.2 \\
\hline \multicolumn{8}{|l|}{ Water solvent } \\
\hline $2 \mathrm{~m}-\mathrm{NO}_{2}$ & -34.4 & -42.9 & -0.563 & 0.221 & 2.070 & 2.350 & 27.6 \\
\hline om- $\mathrm{NO}_{2}$ & -31.4 & -44.1 & -0.546 & 0.210 & 2.092 & 2.339 & 27.5 \\
\hline op- $\mathrm{NO}_{2}$ & -31.1 & -44.1 & -0.546 & 0.198 & 2.088 & 2.339 & 27.7 \\
\hline $2 \mathrm{~m}-\mathrm{NO}_{2}$ (estimated) & -33.6 & -43.7 & -0.556 & 0.219 & 2.082 & 2.336 & 28,7 \\
\hline om- $\mathrm{NO}_{2}$ (estimated) & -30.2 & -44.8 & -0.540 & 0.199 & 2.099 & 2.324 & 28,6 \\
\hline op- $\mathrm{NO}_{2}$ (estimated) & -31.2 & -44.3 & -0.544 & 0.195 & 2.087 & 2.331 & 27,9 \\
\hline $2 \mathrm{~m}-\mathrm{NO}_{2}$ (rel. err.) & 8.1 & 16.0 & 16.1 & 3.0 & 41.2 & 42.4 & 39,6 \\
\hline om- $\mathrm{NO}_{2}$ (rel. err.) & 9.0 & 11.3 & 9.6 & 13.0 & 15.7 & 33.9 & 39,8 \\
\hline op- $\mathrm{NO}_{2}$ (rel. err.) & 0.3 & 4.8 & 2.9 & 3.6 & 2.1 & 22.4 & 7,3 \\
\hline $2 \mathrm{~m}-\mathrm{NO}_{2}$ (abs. err.) & 0.8 & 0.8 & 0.007 & 0.002 & 0.012 & 0.014 & 1,1 \\
\hline om- $\mathrm{NO}_{2}$ (abs. err.) & 1.2 & 0.7 & 0.005 & 0.011 & 0.007 & 0.016 & 1,1 \\
\hline op- $\mathrm{NO}_{2}$ (abs. err.) & 0.0 & 0.3 & 0.002 & 0.003 & 0.001 & 0.009 & 0.1 \\
\hline
\end{tabular}


Table S12: Complexes with poly-NO $\mathbf{N O}_{2}$ ligands: calculated $X_{c}$ and estimated $X_{e}$ values of binding energies (in $\mathrm{kcal} / \mathrm{mol}$ ), ligand charges (in e), bond lengths of $\mathbf{X}-\mathbf{P}$ structures for polysubstituted complexes. Relative and absolute errors are also shown. Estimated values were obtained from Equation 2; relative errors from the ratio $\frac{\left|X_{c}-X_{e}\right| * 100 \%}{\left|X_{e}-X_{H}\right|}$, where $X_{H}$ are the values for the H-R complex; absolute errors represent the difference $\left|X_{c}-X_{e}\right|$.

\begin{tabular}{|c|c|c|c|c|c|c|}
\hline & $\Delta E_{\text {bind }}^{\text {pyr } X}$ & $\Delta E_{\text {bind }}^{w}$ & $\mathrm{q}(\mathrm{pyrX})$ & $\mathrm{q}(\mathrm{w})$ & Pt-N $\mathrm{N}_{\mathrm{pyr} X}$ & $\mathrm{Pt}-\mathrm{O}_{\mathrm{w}}$ \\
\hline \multicolumn{7}{|l|}{ Gas phase } \\
\hline $2 \mathrm{~m}-\mathrm{NO}_{2}$ & -86.2 & -50.1 & 0.386 & 0.188 & 2.021 & 2.123 \\
\hline om- $\mathrm{NO}_{2}$ & -89.6 & -51.3 & 0.355 & 0.196 & 2.042 & 2.111 \\
\hline op- $\mathrm{NO}_{2}$ & -89.0 & -51.3 & 0.354 & 0.195 & 2.041 & 2.112 \\
\hline $2 \mathrm{~m}-\mathrm{NO}_{2}$ (estimated) & -84.2 & -50.4 & 0.384 & 0.188 & 2.021 & 2.124 \\
\hline om- $\mathrm{NO}_{2}$ (estimated) & -87.6 & -51.6 & 0.349 & 0.197 & 2.043 & 2.109 \\
\hline op- $\mathrm{NO}_{2}$ (estimated) & -87.0 & -51.6 & 0.349 & 0.196 & 2.041 & 2.108 \\
\hline $2 \mathrm{~m}-\mathrm{NO}_{2}$ (rel. err.) & 6.4 & 7.6 & 5.9 & 1.7 & 1.3 & 5.7 \\
\hline om- $\mathrm{NO}_{2}$ (rel. err.) & 7.5 & 5.1 & 8.5 & 4.2 & 2.9 & 5.9 \\
\hline op- $\mathrm{NO}_{2}$ (rel. err.) & 7.4 & 6.8 & 6.8 & 5.5 & 1.4 & 11.4 \\
\hline $2 \mathrm{~m}-\mathrm{NO}_{2}$ (abs. err.) & 1.9 & 0.3 & 0.002 & 0.000 & 0.000 & 0.001 \\
\hline om- $\mathrm{NO}_{2}$ (abs. err.) & 2.0 & 0.2 & 0.006 & 0.001 & 0.001 & 0.002 \\
\hline op- $\mathrm{NO}_{2}$ (abs. err.) & 2.0 & 0.3 & 0.005 & 0.001 & 0.000 & 0.003 \\
\hline \multicolumn{7}{|l|}{ Water solvent } \\
\hline $2 \mathrm{~m}-\mathrm{NO}_{2}$ & -42.7 & -22.1 & 0.298 & 0.210 & 2.019 & 2.105 \\
\hline om- $\mathrm{NO}_{2}$ & -38.9 & -22.4 & 0.282 & 0.218 & 2.039 & 2.093 \\
\hline op- $\mathrm{NO}_{2}$ & -39.2 & -22.4 & 0.277 & 0.216 & 2.033 & 2.099 \\
\hline $2 \mathrm{~m}-\mathrm{NO}_{2}$ (estimated) & -42.8 & -21.6 & 0.298 & 0.208 & 2.017 & 2.111 \\
\hline om- $\mathrm{NO}_{2}$ (estimated) & -38.4 & -22.3 & 0.277 & 0.217 & 2.037 & 2.094 \\
\hline op- $\mathrm{NO}_{2}$ (estimated) & -39.0 & -22.7 & 0.271 & 0.218 & 2.034 & 2.089 \\
\hline $2 \mathrm{~m}-\mathrm{NO}_{2}$ (rel. err.) & 0.7 & 47.7 & 0.6 & 13.9 & 22.0 & 83.2 \\
\hline om- $\mathrm{NO}_{2}$ (rel. err.) & 3.0 & 8.0 & 6.6 & 1.1 & 8.9 & 6.8 \\
\hline op- $\mathrm{NO}_{2}$ (rel. err.) & 1.2 & 9.7 & 6.4 & 10.8 & 3.9 & 37.4 \\
\hline $2 \mathrm{~m}-\mathrm{NO}_{2}$ (abs. err.) & 0.1 & 0.5 & 0.000 & 0.002 & 0.001 & 0.006 \\
\hline om- $\mathrm{NO}_{2}$ (abs. err.) & 0.5 & 0.1 & 0.005 & 0.000 & 0.002 & 0.002 \\
\hline op- $\mathrm{NO}_{2}$ (abs. err.) & 0.2 & 0.2 & 0.005 & 0.002 & 0.001 & 0.011 \\
\hline
\end{tabular}




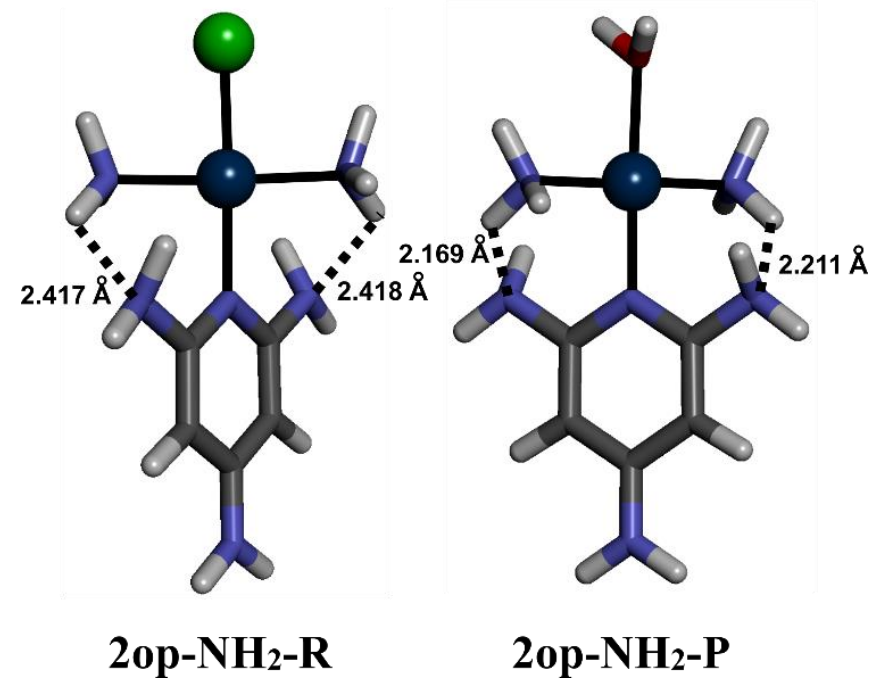

Figure S12: Stabilizing H-bonds in the 2op- $\mathbf{N H}_{\mathbf{2}}-\mathbf{R}$ and 2op- $\mathbf{N H}_{2}-\mathbf{P}$ structures.

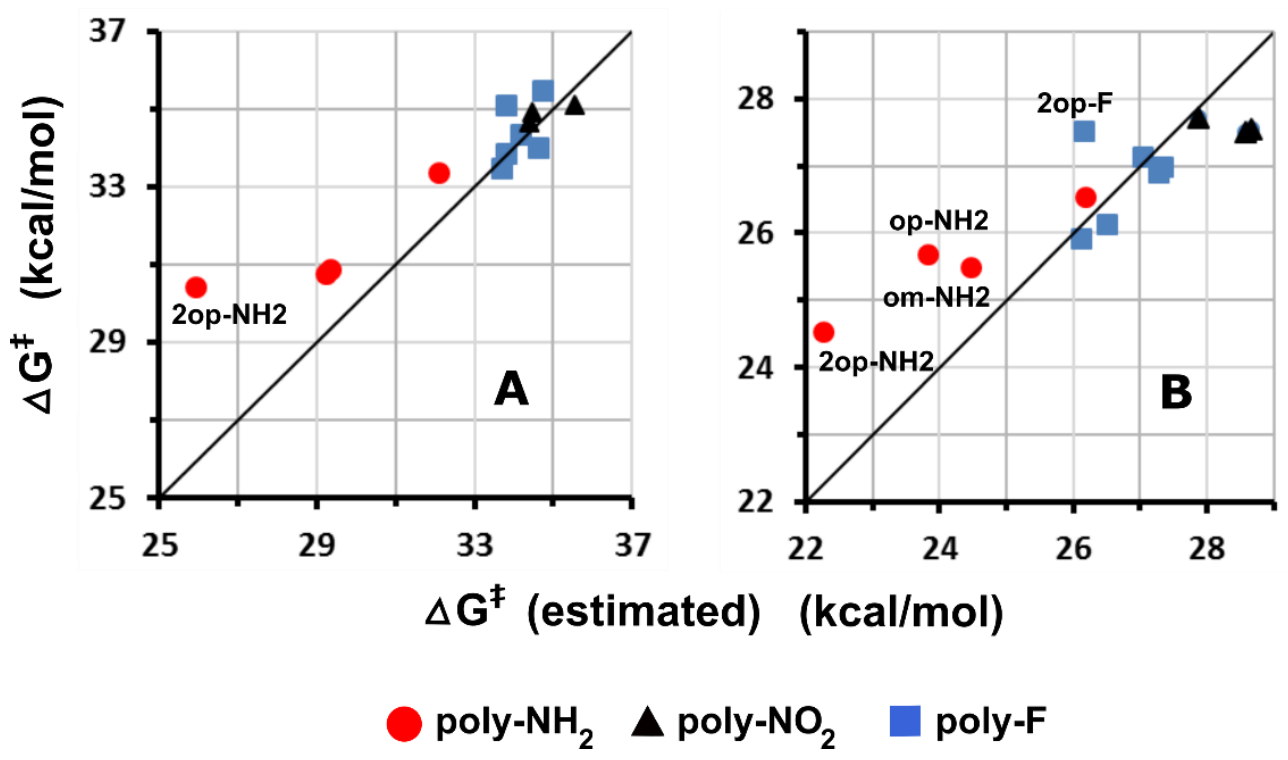

Figure S13: Plots of calculated vs. estimated (Equation 2) values of $\Delta G^{\ddagger}$ activation free energies for complexes with poly-substituted ligands in the gas phase (panel A) and the water solvent (panel B). The solid line represents equality of the two values. 

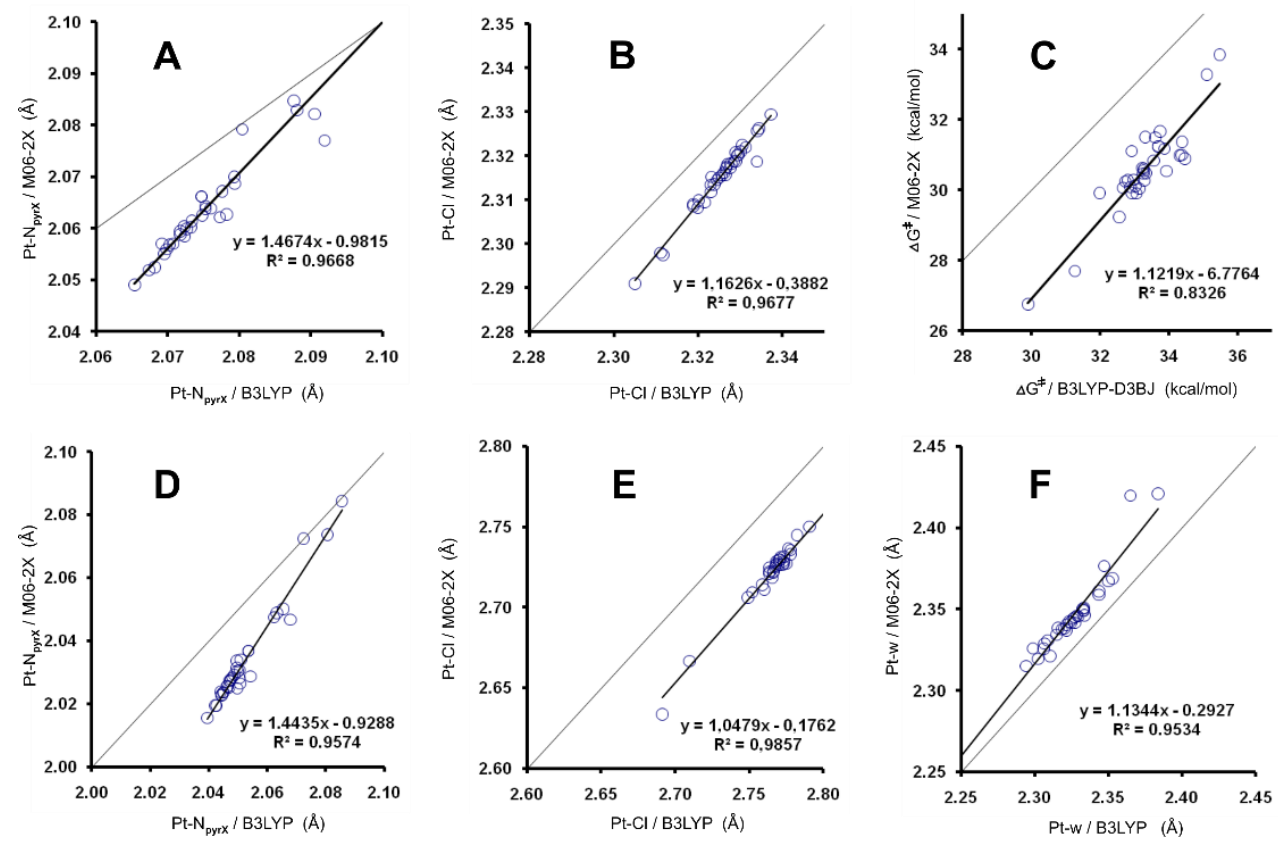

Figure S14: Gas phase calculations: the correlation between B3LYP/BS1 and M06-2X/BS1 bond lengths for X-R_w (panels A, B) and X-TS (panels D, E, F) structures. The panel C shows the correlation between B3LYP-D3BJ//BS2//B3LYP/BS1 and M06-2X/BS2//M062X/BS1 activation Gibbs free energies.
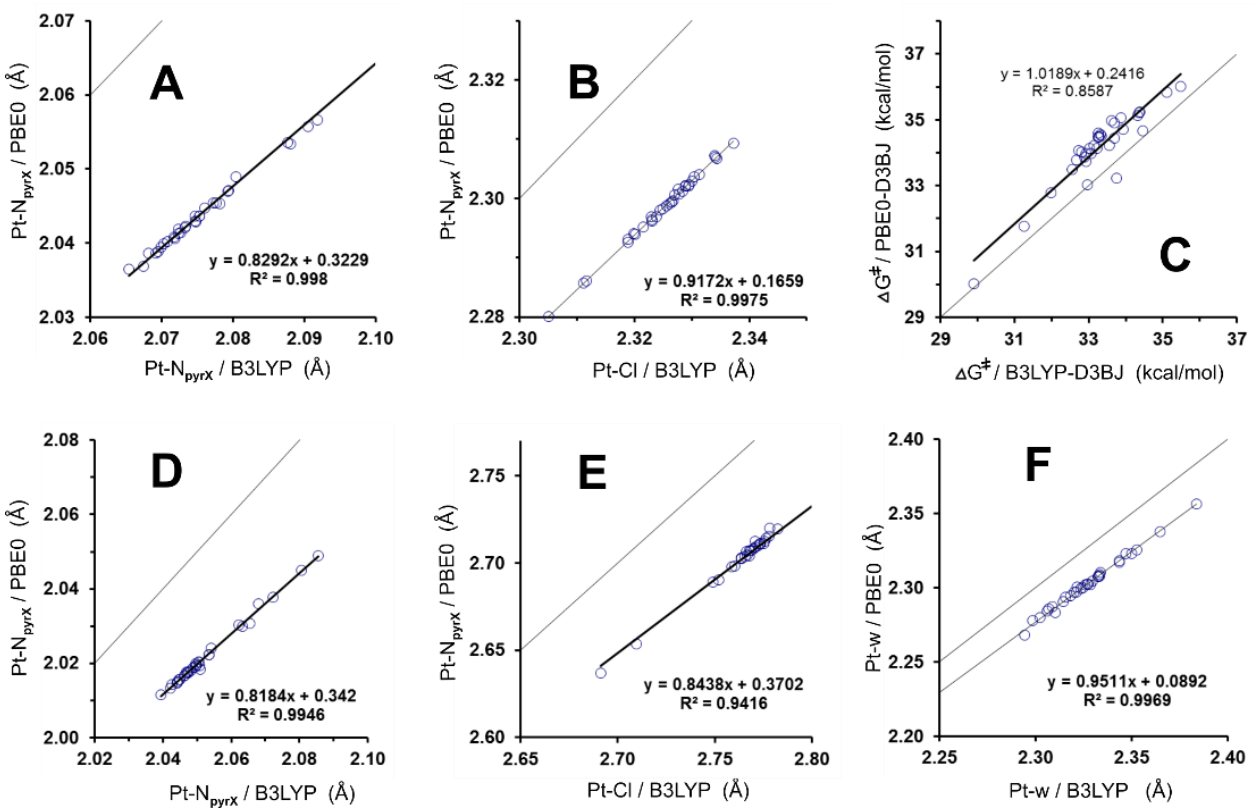

Figure S15: Gas phase calculations: the correlation between B3LYP/BS1 and PBE0/BS1 bond lengths for X-R_w (panels A, B) and X-TS (panels D, E, F) structures. The panel C shows the correlation between B3LYP-D3BJ//BS2//B3LYP/BS1 and PBE0D3BJ/BS2//PBE0/BS1 activation Gibbs free energies. 

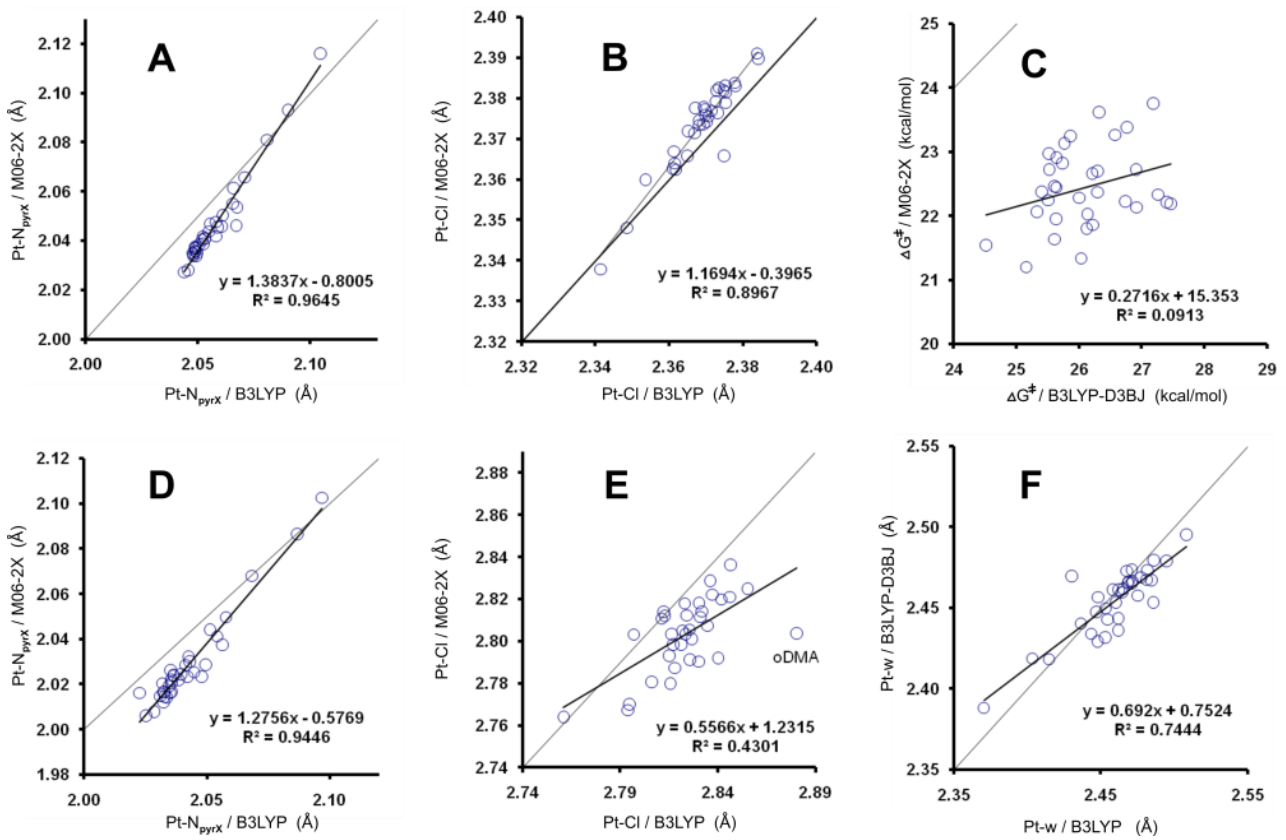

Figure S16: PCM water solvent calculations: the correlation between B3LYP/BS1 and M062X/BS1 bond lengths for X-R_w (panels A, B) and X-TS (panels D, E, F) structures. The panel $\mathrm{C}$ shows the correlation between B3LYP-D3BJ//BS2//B3LYP/BS1 and M062X/BS2//M06-2X/BS1 activation Gibbs free energies.

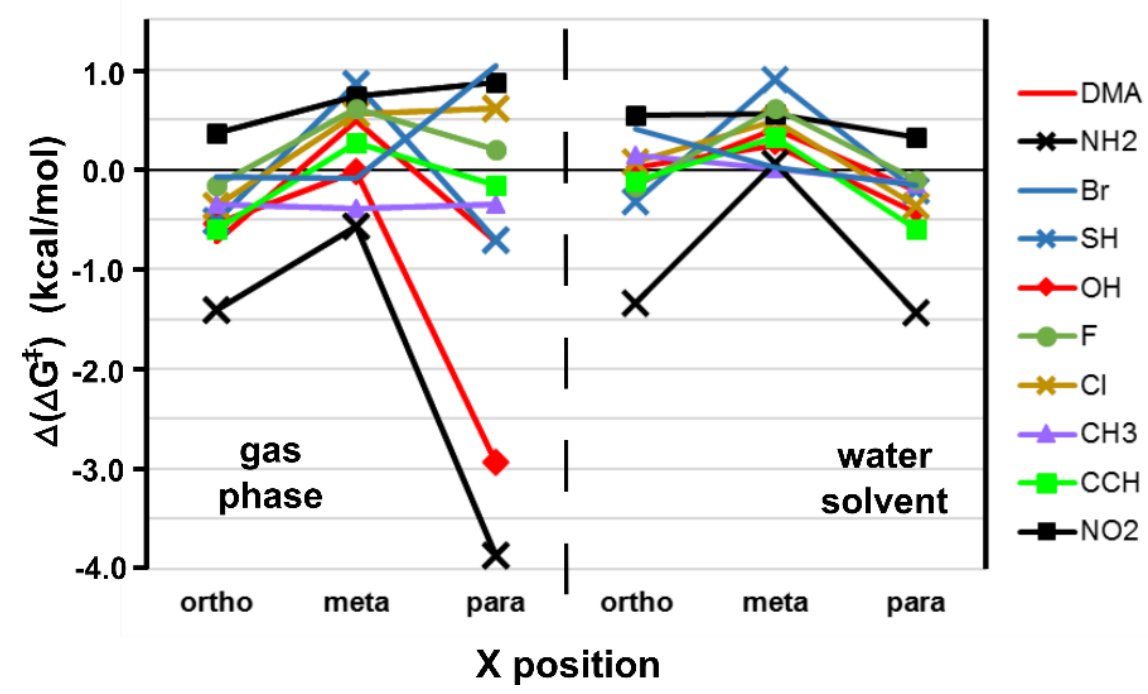

Figure S17: M06-2X/BS2//M06-2X/BS1 calculations: the dependence of $\Delta\left(\Delta \mathrm{G}^{\dot{+}}\right)$ values of the hydration reactions of the trans- $\left[\mathrm{Pt}\left(\mathrm{NH}_{3}\right)_{2}(\mathrm{pyrX}) \mathrm{Cl}\right]^{+}$complexes on the nature and the position of the $\mathrm{X}$ substituent in the gas phase and in the water solvent (cf. Figure 6). $\Delta\left(\Delta \mathrm{G}^{\ddagger}\right)$ were calculated with respect to the reference values $(30.6 \mathrm{kcal} / \mathrm{mol}$ and $22.5 \mathrm{kcal} / \mathrm{mol}$ in the gas and water solvent, respectively) determined for the $\mathrm{X}=\mathrm{H}$ pathway. 